\title{
Tabularia
}

\section{Un exemple de comptabilité hospitalière : les comptes de l'hôtel-Dieu Saint-Thomas d'Argentan (1402-1499)}

An example of hospital accounting: the accounts of the Hospital of Saint-Thomas d'Argentan (1402-1499)

\section{Marie-Anne Moulin}

\section{OpenEdition Journals}

Édition électronique

URL : http://journals.openedition.org/tabularia/1252

DOI : 10.4000/tabularia.1252

ISSN : 1630-7364

Éditeur :

CRAHAM - Centre Michel de Boüard, Presses universitaires de Caen

\section{Référence électronique}

Marie-Anne Moulin, « Un exemple de comptabilité hospitalière : les comptes de l'hôtel-Dieu Saint-

Thomas d'Argentan (1402-1499) », Tabularia [En ligne], Les sources comptables, méthodologie, critique et édition, mis en ligne le 15 décembre 2010, consulté le 30 avril 2019. URL : http://

journals.openedition.org/tabularia/1252; DOI : 10.4000/tabularia.1252

Ce document a été généré automatiquement le 30 avril 2019

CRAHAM - Centre Michel de Boüard 


\section{Un exemple de comptabilité hospitalière : les comptes de l'hôtel- Dieu Saint-Thomas d'Argentan (1402-1499)}

An example of hospital accounting: the accounts of the Hospital of Saint-Thomas d'Argentan (1402-1499)

\section{Marie-Anne Moulin}

Fondé au XII ${ }^{e}$ siècle dans le faubourg Est de la ville naissante d'Argentan, l'hôtel-Dieu de la Trinité puis Saint-Thomas est né d'une initiative bourgeoise, comme se plaisent à le rappeler les actes médiévaux le concernant ${ }^{1}$. Dès son origine, le lieu a pour vocation d'accueillir les femmes enceintes et les jeunes accouchées, les malades et les «pauvres passants ", les enfants abandonnés, les orphelins et les enfants placés par décision de justice, les personnes âgées et tout individu lui faisant don de sa personne ${ }^{2}$. Il fait ainsi office de maternité, d'hôpital, d'orphelinat et d'hospice. Pour mener à bien ses missions, l'institution bénéficie rapidement des largesses des rois-ducs puis dès 1204 de la bienveillance des rois de France et des seigneurs d'Argentan. L'affluence de malades, parfois originaires de paroisses distantes de plus de $40 \mathrm{~km}$, lui vaut de nombreuses donations dans un large rayon autour de la ville. Dès le XIV e siècle, l'hôpital apparait donc doté d'un large patrimoine foncier et immeuble ainsi que d'importants revenus - rentes, dons et aumônes perpétuelles. La gestion de cet imposant ensemble de biens et de droits relève de la mission d'un membre de la bourgeoisie urbaine, désigné par ses pairs, le maître et administrateur. Responsable sur ses biens propres de son administration, le maître et administrateur, désigné pour un mandat de trois ans renouvelable, doit présenter un bilan annuel de ses activités aux représentants du groupe bourgeois. Dès la fin du XIV siècle, ce bilan prend la forme de comptes écrits. C'est une partie de cette comptabilité annuelle qui constitue l'élément essentiel du fonds de l'hôtel-Dieu SaintThomas, aujourd'hui conservé aux Archives départementales de l'Orne. Pour le Moyen Âge, la comptabilité débute en 1402, s'achève en 1499 et regroupe un total de 23 comptes 
riches d'enseignements tant sur la constitution d'une comptabilité au Moyen Âge que sur l'administration hospitalière et l'hôpital lui-même.

\section{Présentation des comptes et description matérielle des documents}

\section{Caractérisation de la série}

2 C'est un concile tenu à Arles en 1260 qui prescrit la tenue et la reddition de comptes hospitaliers réguliers; cette obligation est bien suivie en France dès le début du XIV ${ }^{\mathrm{e}}$ siècle et l'hôtel-Dieu d'Argentan ne fait pas exception ${ }^{3}$. Les premières traces de comptes réguliers datent de 1374. À la suite de l'examen de l'ensemble de la comptabilité de Nicole Le Petit, maître et administrateur de Saint-Thomas, il apparaît que certains tenants de rente restent redevables de sommes importantes envers l'administration hospitalière. Le maître et administrateur est considéré responsable du défaut de paiement à l'issue de cette vérification : «c'est à savoir que tant pour la somme de 9 vingt livres tournois en quoi les deffuncts estoient demeurés en la dette envers le dit maistre [Nicole Le Petit] par la fin de tous ses comptes tant de recette que de mise $»^{4}$. En 1397, un autre acte démontre bien d'une part la tenue régulière de comptes et d'autre part l'habituel contrôle des comptes avant que le maître ne soit déclaré quitte de son administration ${ }^{5}$. Cependant, aucun compte antérieur à l'année 1402-1403 n'a été conservé.

L'essentiel de la série comptable médiévale aujourd'hui archivée couvre la seconde moitié $\mathrm{du} \mathrm{XV}^{\mathrm{e}}$ siècle, bien que deux comptes du début du siècle aient été conservés. Ces deux comptes ne sont ni consécutifs, ni complets ${ }^{6}$. La série existante s'interrompt en 1406 et ne reprend qu'en 1453. Tous les comptes de la seconde moitié du XVe siècle n'ont pas été préservés mais la période présente de belles successions de comptes, notamment dans les années 1450 (4 comptes), dans les années 1470 ( 6 comptes) et dans les années 1480-1490 avec 8 comptes (tableau 1). L'obligation de tenue des comptes ne s'interrompt pas à la fin de l'époque médiévale et la série moderne est d'ailleurs très dense. Quatre-vingt-neuf comptes sont conservés pour le XVI ${ }^{e}$ siècle, 84 pour le XVII siècle, 12 pour le début du XVIII ${ }^{\mathrm{e}}$ siècle et la série est ininterrompue de 1723 à $1790^{7}$.

La question se pose donc de savoir pourquoi une partie de cette importante documentation médiévale ne nous est pas parvenue et l'examen des sources modernes permet de répondre partiellement à cette problématique. L'habitude de tenir des comptes dès la fin du XIV ${ }^{e}$ siècle a bien été démontrée mais n'est connue que par des copies d'actes de constitution de maitres et administrateurs ou par des actes de quittance de leur gestion. Les comptes de la première moitié du XIV ${ }^{e}$ siècle auraient pu être mis à mal par l'attaque anglaise menée contre la ville en 1356. Michel de Courteilles, dans son histoire d'Argentan, datée de 1692-1693, mentionne la perte d'une partie des titres de l'hôpital lors de cette chevauchée ${ }^{8}$. Il ne reste cependant pas certain qu'une comptabilité ait été rédigée avant 1374. Les inventaires modernes des titres de la maison-Dieu signalent la conservation d'actes médiévaux mais aucun n'indique l'existence de comptabilité dans les archives hospitalières de l'époque. Seules les comptabilités récentes y sont en général mentionnées ${ }^{9}$. Il apparaît cependant qu'un nombre plus important de comptes médiévaux existe encore à la fin du XVI ${ }^{e}$ siècle. Des passages de plusieurs comptes médiévaux sont transcris à l'époque moderne soit par les responsables de Saint-Thomas soit par d'autres institutions ou personnes privées, pour être utilisés comme preuves au cours de procès. 
Les comptes médiévaux sont ainsi retranscrits jusqu'au XIX ${ }^{\mathrm{e}}$ siècle à des fins judiciaires et des extraits en apparaissent également dans les titres de propriété de l'hôtel-Dieu pour justifier de droits ou de la jouissance de biens ${ }^{10}$. Le recours aux comptes médiévaux ne se fait pas uniquement à cette fin mais aussi dans un but de gestion interne et de répartition des taches et des rôles au sein de l'hôtel-Dieu ${ }^{11}$. Ces copies partielles permettent de certifier la conservation d'archives comptables plus conséquentes à l'époque moderne. Les comptes de 1407-1408 et de 1417-1418, aujourd'hui disparus, ont été exploités et en partie recopiés lors d'un procès opposant l'abbaye Notre-Dame d'Almenêches à plusieurs familles bourgeoises d'Argentan en $1631^{12}$. Un historiographe local, lors de la rédaction de son ouvrage au XVII e siècle, appuie sa démonstration sur les comptes de 1445-1448, aujourd'hui manquants ${ }^{13}$. Il est possible que l'extraction de certains comptes de la série à des fins judiciaires ou de recherches historiques explique leur disparition. Il est aussi envisageable que les archives hospitalières (sauf celles visées en 1631), à l'instar des archives des dominicains de la ville, aient souffert de destructions lors des guerres de religion, particulièrement violentes dans la région ${ }^{14}$. Des destructions des fonds d'archives pendant la période révolutionnaire paraissent plus hypothétiques au vu de la très bonne conservation des titres récents de la maison-Dieu. Lorsque l'inventaire sommaire de la série $\mathrm{H}$ des Archives départementales de l'Orne est dressé et publié en 1899, l'archiviste Louis Duval ne dispose déjà plus de l'intégralité des comptabilités médiévales. Son travail se fonde sur des catalogues, des copies et des extraits de comptes, peut-être établis à l'occasion. Les comptes ou extraits de comptes qu'il examine alors sont ceux de 1402-1403, 1405-1406 et 1456-1489 et il n'est fait référence à aucun autre compte médiéval dans son travail. Les comptes hospitaliers conservés à la fin du XIX ${ }^{\mathrm{e}}$ siècle sont donc ceux qui ont été versés aux départementales de l'Orne en $1965^{15}$.

5 L'absence d'une comptabilité hospitalière médiévale complète n'est pas uniquement liée aux aléas de la conservation. Il faut aussi signaler le non respect ponctuel, à l'époque médiévale, de l'obligation de tenue des comptes hospitaliers. Aucun acte de gestion comptable définitif n'a été rédigé entre 1417, début de l'occupation anglaise de la Normandie, et 1435. L'hôtel-Dieu est alors considéré par les autorités anglaises comme un bénéfice religieux, confié à un ecclésiastique désigné par le roi d'Angleterre, et non plus comme une institution bourgeoise. Les gouvernants se désintéressent de sa gestion et ne sont apparemment pas tenus à la reddition de comptes annuels, ni à leur présentation devant le souverain ou son représentant. Cependant, l'existence d'extraits de la recette des rentes des années 1431-1433, insérés dans le premier registre de comptabilité, laisse supposer un suivi relatif de la gestion hospitalière mais ne permet pas de confirmer la tenue de comptes définitifs. Un relâchement dans la gestion des biens et dans le suivi comptable a pu être repéré au même moment dans d'autres institutions argentanaises comme, par exemple, la fabrique de Saint-Germain d'Argentan. Pour la période de l'occupation les comptes en apparaissent moins soignés et moins ordonnés ${ }^{16}$.

6 Des comptes sont a priori tenus à partir de 1435 par le prêtre anglais Huntyngton, désigné maître et administrateur par les bourgeois. Mais son refus de présenter des justificatifs de son administration, au prétexte qu'il tient l'hôpital du roi d'Angleterre, rend crédible l'hypothèse d'une absence totale de comptabilité hospitalière définitive sous son autorité. Aucune trace d'une comptabilité entre 1433 et 1445 n'a pu être trouvée dans l'historiographie locale ni dans les sources archivistiques. L'antagonisme naissant entre le groupe bourgeois et le maître de l'hôtel-Dieu a très bien pu, également, entraîner une interruption de la comptabilité initialement rédigée. Il est aussi possible que la 
comptabilité des années 1435 n'ait pas été conservée ultérieurement et cette hypothèse est d'autant plus crédible qu'il apparaît que des comptes ont bien été composés entre 1445-1448 sous l'administration Hungtyngton ${ }^{17}$. Par l'élimination de toute trace écrite de sa gestion, les bourgeois auraient alors voulu faire oublier leur choix d'un mauvais administrateur et minimiser leur responsabilité dans la ruine partielle de l'établissement ${ }^{18}$.

7 Le rétablissement des finances de Saint-Thomas qui fait suite à ce conflit débute avec le choix de deux administrateurs provisoires en octobre 1448, solution prescrite par un jugement du Parlement de Normandie, auquel les deux parties ont fait appel pour établir leur bon droit ${ }^{19}$. Il est plus que probable que l'une des premières tâches de ces deux hommes a été l'examen attentif des comptes subsistants de Huntyngton et l'établissement d'un compte provisoire, permettant de faire le point sur l'état des finances de la maison. Mais les archives hospitalières ne portent pas trace d'une telle inspection et ne recèlent aucune comptabilité transitoire pour cette date. Bien que le premier compte conservé après l'occupation anglaise date de 1453 , la nomination d'un nouveau titulaire au poste de maître a de fait nécessité la tenue d'une nouvelle comptabilité à partir de 1449, d'autant plus que le litige opposant les bourgeois et le précédent administrateur portait sur l'absence de justificatifs comptables de sa gestion.

8 Sur les 23 comptes originaux qui nous sont parvenus, seuls onze comptes - un douzième peut-être - ont été intégralement conservés, sans interruption dans le texte ni modification de leur mise en page depuis l'époque médiévale. Ces 23 comptes sont inégalement reliés en dix registres, contenant entre un compte unique et quatre comptes classés dans l'ordre chronologique. Les aléas de la conservation et les dommages causés aux archives depuis le Moyen Âge expliquent que certains comptes soient aujourd'hui amputés de certaines parties, endommagés ou incomplets. Les lacunes sont plus ou moins importantes, de quelques pages à des chapitres entiers voire à une quasi totalité du compte (tableau 1).

\section{Description des comptes conservés}

9 Les comptes sont, à partir de 1454, tenus sur papier et seuls les trois exercices comptables définitifs du premier volume ont été rédigés sur parchemin, conformément à de nombreuses séries comptables de début du siècle. L'habitude d'une rédaction sur parchemin constatée pour les comptes de 1402-1403 et 1405-1406 se poursuit, notamment dans les années 1407-1408 et 1416-1417, jusqu'en 1453 pour être abandonnée en $1454^{20}$. L'usage tardif du parchemin apparait d'autant plus surprenant que les pièces comptables annexes - journaux des dépenses, journaux de la recette - sont déjà consignées sur papier dans les années 1430 comme l'attestent les fragments de 1431-1433 inclus dans le premier registre comptable. Cependant, l'hôtel-Dieu continue à inscrire les actes majeurs - cessions ou achats de terrains et de biens, contrats de réception de condonnés, actes de désignation des maîtres - sur parchemin jusqu'au début du XVI ${ }^{\mathrm{e}}$ siècle, période à laquelle l'usage du papier se généralise lentement pour l'ensemble des écrits hospitaliers ${ }^{21}$.

10 Les trois comptes regroupés dans le premier registre sont rédigés sur un parchemin très fin fabriqué sur place avec des peaux des réserves de l'hôtel-Dieu ${ }^{22}$. Chacun de ces comptes comporte plusieurs cahiers reliés, rassemblés par une couverture de peau. L'emploi de parchemin démontre la volonté de l'administrateur hospitalier d'inscrire sa 
comptabilité dans la durée en en assurant la bonne conservation mais prouve aussi une certaine ostentation du groupe bourgeois dans sa gestion de l'établissement.

11 C'est un papier identique qui a servi à la rédaction de tous les comptes à partir de 1454. Il se caractérise par des dimensions importantes et par un filigrane de licorne simple apposé au centre de chaque feuillet. Ce papier est identique à celui utilisé à la même époque pour la tenue des divers cahiers et journaux des recettes et des dépenses, de dimensions similaires et sur lesquels se repère également le filigrane de licorne. La qualité de ce papier doit être soulignée. C'est un papier épais et solide qui confirme le souci des maîtres de présenter une belle comptabilité. Il faut préciser que l'utilisation du papier pour la tenue de registres comptables n'est pas encore la norme dans la région dans les années 1440-1450 et le choix de ce support à l'hôtel-Dieu démontre également un souci d'économie dans la confection des comptes ${ }^{23}$.

12 La constance du format du papier et la présence d'un unique filigrane atteste une fourniture auprès d'un seul fabricant. Ces éléments plaident en faveur d'une fourniture très locale, bien qu'aucun moulin à papier n'ait été localisé dans la ville et ses alentours au XVe siècle ${ }^{24}$. L'identité du marchand chargé d'approvisionner l'hôtel-Dieu en papier est connue dans les années 1450, il s'agit de Thibault de La Lande puis de sa veuve qui reprend son activité dans les années $1460^{25}$.

13 Les filigranes de licorne simple sont très répandus en Europe de l'Ouest et plus particulièrement en Normandie au Moyen Âge. Cependant aucun filigrane identique à celui des comptes de Saint-Thomas n'a été répertorié dans le recensement établi par Briquet au $\mathrm{XIX}^{\mathrm{e}}$ siècle ${ }^{26}$. Le filigrane argentanais se distingue des types régionaux similaires par ses dimensions très inférieures. Longue de $88 \mathrm{~mm}$ et haute de $53 \mathrm{~mm}$, la licorne est placée au centre de chaque feuillet ${ }^{27}$. Un filigrane semblable orne le papier utilisé pour la comptabilité paroissiale de Saint-Germain ${ }^{28}$. Par le graphisme, ce filigrane se rapproche de filigranes relevés sur des papiers utilisés à Almenêches, Silly ou encore Alençon ${ }^{29}$.

D'autres filigranes ornent les couvertures papier de plusieurs reliures de cahiers hospitaliers médiévaux: blasons fleuris, blasons fleurdelisés, initiales MC et soleil (?). Aucun d'entre eux n'est non plus catalogué dans les travaux de Briquet, qui permettent cependant de les classifier dans des types plus généraux. Le blason fleuri, visible sur les couvertures du compte de 1495-1496, appartient au type des « écus à trois fleurs posées deux et une » recensé par Briquet. Tous les filigranes de ce type datent du XVI ${ }^{e}$ siècle et aucun ne provient de Basse-Normandie ${ }^{30}$. Les «trois fleurs de lis deux et une posées dans un écu couronné ", présentes dans le compte de 1473-1474, ont bien été observées pour le $\mathrm{XV}^{\mathrm{e}}$ siècle $^{31}$. Les lettres initiales, observables sur la couverture du compte de 1487-1488, sont courantes et pourraient correspondre aux initiales du fabricant. Les deux cercles inscrits l'un dans l'autre et reliés par des rayons ondoyants à l'extérieur peuvent être assimilés aux filigranes de soleil, par ailleurs assez variés, datés du $\mathrm{XV}^{\mathrm{e}}$ siècle $^{32}$. Ce filigrane apparaît au milieu du compte intermédiaire de 1479, à la page 85.

Plusieurs hypothèses peuvent expliquer l'utilisation d'un papier différent pour couvrir les comptes médiévaux. Soit il s'agit d'une couverture réalisée avant ou immédiatement après la reddition des comptes et on peut alors y voir la volonté de couvrir les cahiers avec un papier de qualité supérieure, plus résistant. Mais il faut signaler ici que les achats de papier sont mentionnés dans les comptes dans une seule et même rubrique, sans distinction de provenance, ce qui ne permet pas de distinguer deux qualités de papier. Soit il s'agit de couvertures postérieures, de l'époque moderne, ce que tendrait à prouver 
l'utilisation d'un filigrane fleurdelisé, datable du XVI siècle. La couverture d'autres comptes médiévaux locaux avec du parchemin va elle aussi dans le sens d'une reliure et d'une couverture des cahiers plus tardive.

Tous les comptes se caractérisent tout au long du $\mathrm{XV}^{\mathrm{e}}$ siècle par des dimensions importantes, dont les larges pages permettent de mettre en valeur les différents chapitres. Ces dimensions distinguent la comptabilité de Saint-Thomas des comptabilités locales contemporaines, au format plus réduit, notamment de celle de Saint-Germain (H $215 \mathrm{~mm}$ x L $145 \mathrm{~mm}$ ), ou des formats plus imposants habituellement relevés pour les papiers français de la fin du XV $\mathrm{XV}^{\mathrm{e}}$ siècle $(\mathrm{H} 430 \mathrm{~mm} \text { x L } 305 \mathrm{~mm})^{33}$. Cependant, ce format n'est pas inédit dans la région et peut être comparé aux dimensions des registres de la seigneurie de Montgommery, conservés aux Archives départementales de l'Orne ${ }^{34}$.

Le nombre de cahiers par registre et par compte est variable. La forme de ces cahiers est comparable à ceux de Saint-Germain ou de Silly, conservés sous leur forme initiale (tableau 2).

La mise en page, quelle que soit la nature du compte produit, répond à quelques règles toujours observées. La première de ces règles est le traçage de marges, présentes des deux côtés de chaque page dès le début de la série comptable. La marge extérieure ainsi délimitée reçoit les sommes de petits paragraphes et les corrections avant l'audition et la réception du compte, mais aussi les demandes de précision et les commentaires des auditeurs, écrits à mesure que l'audition se déroule. La marge intérieure de la page ne porte habituellement pas d'écriture mais permet un réel confort de lecture et démontre que dès la rédaction, une reliure des cahiers du même compte était soit déjà réalisée soit envisagée. Dans les comptes de 1405 à 1499, la mise en page se fait sur une seule colonne qui occupe l'entièreté de l'espace délimité par les marges alors que dans le compte de 1402-1403 le texte est réparti sur deux colonnes de taille égale. La présence de réglures, tracées à la pointe sèche, dans l'intégralité de la comptabilité démontre un soin constant apporté à la rédaction et à la présentation des comptes.

19 La page de garde de chaque compte s'ouvre sur un paragraphe stipulant les dates d'entrée et de fin du compte, le nom du maître en charge et porte rappel de son mandat (fig. 1 à 4 ). Cette introduction au compte s'orne habituellement d'une lettrine initiale très soignée et dans le compte de 1484-1485, c'est une miniature représentant l'archevêque Thomas Becket qui illustre cette première page (fig. 4).

Quel que soit le soin apporté à la rédaction, la mise en page veille toujours à présenter les grands chapitres, les paragraphes et chaque dépense ou recette de façon nettement séparée du reste du texte. Une volonté de lisibilité et de facilité de lecture y est donc perceptible. La netteté du texte et des données est renforcée par la présence de titres de parties et de petits titres intermédiaires spécifiant le contenu global du paragraphe ou de la partie suivante. Les titres, souvent en capitales et en caractères plus imposants, sont souvent portés en gras pour des repérages plus faciles de données ou de renseignements. Dans tout paragraphe, chaque dépense ou chaque recette est séparée par un interligne supérieur et chaque item est annoncé par un tiret. En cela, la volonté initiale de faire des comptes une source archivistique utilisable à des fins juridiques ou comme référence est très claire.

21 L'ensemble de la série montre une présentation assez aérée qui rend le texte très lisible. Dans les comptes pré-rédigés, la mise en page prévoit de larges espacements entre les 
mots ou groupes de mots ou entre les paragraphes à compléter pour permettre l'ajout de précisions utiles avant l'audition (tableau 3).

Les comptes sont tous rédigés au recto et au verso, en français, mises à part les sommes de fin de chapitre, portées en latin. Bien que les écritures des différents maîtres et scribes soient plus ou moins soignées et que la rédaction leur ait pris plus ou moins de temps et d'attention, il n'en demeure pas moins que tous les comptes restent parfaitement lisibles. Dans leur ensemble, les comptes offrent une écriture et une présentation claire, aérée et soignée. Les comptes dont la tenue est attribuable au maitre en fonction ne font pas exception et seul l'un d'eux, le compte de 1456-1457 écrit conjointement avec un scribe, se distingue par une présentation et une écriture grossières. A contrario, l'emploi d'un scribe s'il est souvent synonyme d'une belle mise en page et d'une écriture particulièrement lisible n'est pas toujours gage de qualité, pour exemple ce même compte de 1456-1457 ou encore celui de 1457-1458 où les trois scribes successifs n'ont pas fait montre d'un grand professionnalisme. Il apparaît donc, au vu de ces données, que l'emploi ou non d'un scribe n'est pas défini par ses qualités de rédacteur mais par la capacité du maître à trouver le temps de rédiger le compte; c'est ce que confirme l'examen de la biographie de l'un de ces scribes, Romain Pigache.

Généralement, les scribes employés à la rédaction des actes comptables hospitaliers sont des inconnus. Leur nom n'est jamais stipulé au moment du paiement de leur travail. Le paragraphe relatif au coût de rédaction et de réalisation d'un compte indique simplement la nature des tâches pour lesquelles ils sont payés, soit qu'il s'agisse de la rédaction du compte seul, soit qu'il s'agisse de la rédaction du compte et des autres documents comptables propres à établir le compte définitif. Le soin apporté aux comptes écrits par des scribes laisse penser qu'il s'agit non seulement de professionnels de l'écrit mais surtout de lettrés dotés de solides compétences comptables et d'une bonne culture juridique. C'est incidemment que le nom d'un scribe nous est parvenu. Pour des raisons inconnues, cet homme, Romain Pigache, est amené à abandonner la rédaction du compte qu'il menait, obligeant ainsi l'hôpital à lui trouver un remplaçant. Ce changement de main fait l'objet d'une précision lorsqu'il se produit, à la page $141 \mathrm{du}$ compte de 1457 : « ysy fault le compte que Romain Pigache n'a pas achevey ce faudra reprendre ysy pour ce que depuis ysy il est pas de l'ordre de l'autre ». Il s'agit ici pour l'administrateur d'expliquer le changement d'écriture tout autant que de justifier d'éventuels frais supplémentaires ou défauts dans la rédaction à venir. Le nom de Romain Pigache permet de dresser un profil des scribes employés à Saint-Thomas. Le choix de Romain Pigache pour la tenue des comptes hospitaliers de 1457 à 1462 est un choix judicieux et ne doit rien au hasard. Ce dernier est un clerc qui accumule les fonctions administratives - procureur du vicomte d'Argentan en 1446-144735 - , et les fonctions comptables - receveur de la vicomté d'Argentan à partir de 1454, receveur d'un impôt urbain en $1452^{36}$, auditeur des comptes de Saint-Germain en tant que procureur des bourgeois en 1462-1463, avant d'être chargé de la rédaction de la comptabilité hospitalière. Il a lors de la rédaction des comptes de Saint-Thomas déjà fait ses preuves en tant que scribe puisqu'il a exercé les fonctions de clerc du vicomte d'Argentan en 1446-1447 ${ }^{37}$. La tenue des comptes hospitaliers n'est qu'une facette de son travail et qu'une étape vers des responsabilités plus importantes qu'il atteint dans les années 1470 - procureur des habitants de la vicomté d'Argentan et d'Exmes aux États de Normandie en $1476^{38}$. Il est par la suite désigné auditeur de plusieurs des comptes qu'il a rédigés $(1458-1459,1460-1461)$ et d'autres comptes hospitaliers $(1454-1455,1479,1484-1485,1486-1487)$. Sa capacité à tenir des comptes et sa 
connaissance de l'institution président au choix des bourgeois dans les années 1478-1479 alors que se déroule l'audition des comptes de Lemesnagier et celle des comptes ultérieurs.

Les comptes tels qu'ils étaient présentés aux bourgeois lors de leur audition au $\mathrm{XV}^{\mathrm{e}}$ siècle, différaient sensiblement de ce qu'ils sont aujourd'hui. Une reliure regroupant les cahiers d'un même compte a bien existé au Moyen Âge et reste, par exemple, visible dans le tome $5 / 2$ ou le tome 7. Au contraire de la reliure de séries comptables régionales contemporaines $^{39}$, il ne s'agit pas ici d'une reliure de parchemin mais d'une reliure de papier épais, dont le filigrane diffère des pages intérieures du compte, comme il a déjà été indiqué. Chaque reliure médiévale rassemble les différents cahiers d'un même compte mais aujourd'hui l'ensemble des vingt trois comptes hospitaliers médiévaux est réuni en dix volumes. Ceux-ci rassemblent un nombre variable de comptes, d'un compte unique à 4 comptes distincts. Les comptes ont été rassemblés et reliés dans leur ordre chronologique et cette reliure est en toute logique intervenue après la disparition de certains comptes, à la fin du XVII ${ }^{e}$ siècle. Le classement des comptabilités hospitalières anciennes prend en compte leur ordre chronologique mais respecte aussi un souci de taille des registres à confectionner de même qu'une volonté de relier ensemble les comptes d'un même maitre. Le dixième volume de la série - le tome 7 - démontre bien cette cohérence puisqu'il inclut les quatre seuls comptes conservés pour l'administration de Gautier Chéradame. La volonté de produire des registres de taille comparable a cependant poussé les autorités hospitalières à réunir dans le premier registre trois comptes d'époques différentes et réalisés sous l'autorité de maitres distincts.

L'assemblage des comptes médiévaux à une époque postérieure à leur rédaction est confirmé par la présence dans l'épaisseur de certaines couvertures d'actes médiévaux plus récents que les comptes qu'ils regroupent. Ainsi, le tome 1 comporte sous la couvrure un acte de Jehan Duval, vicomte d'Argentan, portant sur le droit de justice du maître de l'hôtel-Dieu, Jean Bury, sur certains héritages. Ces deux noms permettent de dater le document des années 1435-1438 ${ }^{40}$. Deux séries d'actes - journaux du maître de Saint-Thomas ? - datables de la fin du $\mathrm{XV}^{\mathrm{e}}$ siècle remplissent pareillement la couverture du tome 6/1.

Plusieurs autres comptabilités locales ont elles aussi fait l'objet d'un assemblage et d'une reliure postérieurs à l'époque médiévale. Les comptes de la vicomté d'Exmes de 1445-1447 ont probablement été pareillement reliés à l'époque moderne ${ }^{41}$ et ceux de la fabrique Saint-Germain d'Argentan l'ont été plus tardivement, au début du XX $\mathrm{XX}^{\mathrm{e}}$ siècle.

Les couvertures des dix volumes peuvent être datées d'une même période, probablement le XVII siècle. En effet, l'existence en 1631, de comptes qui n'ont pas été inclus dans les registres comptables, laisse entendre que la reliure a eu lieu plus tard, alors que ces comptes ont déjà disparu ${ }^{42}$. Elle est réalisée en peau, munie de quatre liens qui, lorsqu'ils sont noués, assurent la fermeture au niveau de la tranche. Des annotations ont d'ailleurs été portées, à la même époque, sur la tranche de chaque volume, signalant les années de comptes qu'il inclut et le nom du ou des maîtres alors en charge. La réalisation de couvertures et l'ajout d'indications sur la tranche semblent avoir été le fait de l'administration hospitalière - souci d'accès facilité à une référence en cas de litige ou de procès ? - ou celui d'un historien argentanais de cette époque. Il s'agit donc de registres factices réalisés à l'époque moderne soit pour mettre de l'ordre dans les archives hospitalières, soit pour l'usage d'historiographes locaux, déjà très actifs à Argentan dès la fin du XVII siècle. Chaque volume regroupe de deux à quatre comptes, pas toujours 
complets. Seul le tome 6, constitué d'une seule année comptable, fait exception. L'assemblage des comptes médiévaux s'est fait dans l'ordre chronologique et a pris en considération les lacunes existantes. L'ordonnancement des comptes médiévaux n'a d'ailleurs pas été perturbé à l'époque de la reliure. La reliure visible aujourd'hui, similaire à celle des journaux de rentes, est faite de belle peau qui, comme le style et la graphie des indications à l'encre portées sur la tranche, plaide en faveur d'une reliure du XVII ${ }^{\mathrm{e}}$ siècle, période à laquelle la ville d'Argentan est d'ailleurs connue pour la qualité de ses cuirs et de sa peausserie. Cette hypothèse est d'autant plus valide que l'ensemble des comptes modernes est pareillement relié. Les informations clairement notées à l'encre sur la tranche de chaque registre démontrent un souci de faciliter l'accès aux archives et une volonté de pouvoir réutiliser les comptabilités anciennes. Les dates et noms indiqués sur les tranches correspondent à une graphie moderne et sont tous d'une même main. Il est très probable que ces indications ont été inscrites au moment de la réalisation de la reliure ce qui semble en indiquer le but : classer, conserver et rendre accessibles les comptes pour des raisons essentiellement juridiques pour pouvoir prouver l'origine de droits et de terres et biens dont jouit l'hôpital. D'autres indications, au crayon à papier, ont également été ajoutées sur les tranches des registres comptables au XIX ${ }^{\mathrm{e}}$ ou au début $\mathrm{du} \mathrm{XX}^{\mathrm{e}}$ siècle. Il s'agit d'une numérotation dans l'ordre chronologique attribuable, soit aux archivistes de l'hôpital, soit à des historiens locaux.

À une époque indéterminée, probablement le $\mathrm{XVI}^{\mathrm{e}}$ siècle, les pages vierges des comptes médiévaux ont fait l'objet d'une récupération et/ou d'une réutilisation. Les feuillets vierges qui séparaient à l'origine les différents comptes et les chapitres des recettes et dépenses ont ainsi été soigneusement découpés ou déchirés ${ }^{43}$. Cependant, le texte d'origine n'est jamais interrompu malgré ces mutilations ${ }^{44}$. Les espaces laissés libres de toute écriture au Moyen Âge ont quant à eux souvent été employés à des exercices d'écriture ${ }^{45}$, à des essais de plume ${ }^{46}$, à la rédaction de brouillons d'actes notariés ${ }^{47}$ ou plus simplement à des petits dessins (tableau 4).

Parmi les 23 comptes, plusieurs ont été foliotés lors de leur rédaction au XV siècle ; sur quatre d'entre eux figure en effet une foliotation en chiffres romains en début de compte, qui s'interrompt après quelques pages ${ }^{48}$, et un seul compte a été totalement foliotée ${ }^{49}$. Cette numérotation médiévale est portée en haut à droite de chaque feuillet. Il est difficile d'expliquer la présence ou l'absence d'une foliotation originale. Si elle correspondait à une volonté d'organiser les archives ou plus ponctuellement à la volonté de rendre l'audition du compte plus aisée, il est alors difficile d'en expliquer l'interruption au bout de quelques pages ou sa totale absence dans la plupart des comptes. Les comptes foliotés au Moyen Âge, de façon partielle ou complète, sont tous des comptes définitifs et sur ces cinq comptes quatre ont été préparés pour l'audition et deux ont été effectivement vérifiés. Il apparaît donc que la foliotation a été réalisée lors de la préparation du compte pour son examen et qu'il s'agit probablement d'une volonté de faciliter l'audition à la fois pour les auditeurs et pour le maître qui présente ses comptes. Une seconde pagination, en chiffres arabes, est venue se superposer sur la foliotation médiévale lorsque celle-ci existait. Tous les registres de comptes portent une pagination similaire datable de la même époque. Cette pagination, continue du début d'un volume à sa fin sans considération du nombre de comptes inclus, est portée avec une encre comparable à celles des annotations des archivistes de la fin du XIX siècle $^{50}$. Cette numérotation systématique des pages de comptes au $\mathrm{XIX}^{\mathrm{e}}$ siècle n'est pas une particularité 
argentanaise, des paginations similaires se retrouvent sur la comptabilité hiémoise et celle de la seigneurie de Montgommery.

\section{Caractères internes des comptes}

\section{Dates d'ouverture et de clôture}

Les dates d'ouverture et de clôture des comptes hospitaliers, toujours indiquées en préambule de ceux-ci, varient au cours du $\mathrm{XV}^{\mathrm{e}}$ siècle. Chaque compte est tenu pour une durée d'un an ainsi que le spécifie le paragraphe d'introduction. D'abord présentés le 21 mai en 1402 , leur reddition se fait le $1^{\text {er }}$ mars en 1408, puis ils débutent au mois de novembre dans les années 1450-1470 puis en juillet à la fin du siècle. Les dates du 21 mai, $\mathrm{du} 1^{\mathrm{er}}$ mars, du 15 novembre, du 30 juin et du 23 juillet ne correspondent à aucun moment liturgique majeur de la vie de l'hôpital, notamment pas à la saint Thomas. Les historiographes locaux se sont toujours accordés pour dire que la date d'entrée des comptes correspondait à la date d'entrée en fonction de chaque maître, ce que semblent confirmer les actes de constitution de maittres dont les copies nous sont parvenues. Cependant, rien n'explique dans ce cas les variations de date au cours du siècle si ce n'est la date fixée par le jugement du Parlement de Normandie en 1449 et le choix d'un changement de date après la mort de Charles Lemesnagier. La date choisie pour la reddition des comptes à partir de 1449, à savoir le 15 novembre, a été fixée non pour des raisons religieuses ou de facilité de gestion, mais a été décrétée par voie de justice. À l'issue du long procès les opposant aux autorités anglaises, les Argentanais obtiennent du Parlement de Normandie la reconnaissance de leur contrôle sur l'hôtel-Dieu et la mise en place de deux maîtres et administrateurs temporaires à sa tête en attendant la désignation d'un titulaire. C'est ce jugement qui instaure la date d'entrée en charge du nouveau maître, donc de fait le changement de date de reddition des comptes ${ }^{51}$.

31 À la mort de Charles Lemesnagier, c'est une décision des représentants des bourgeois qui fixe le nouveau terme de reddition des comptes au 30 juin de chaque année, bien que le dernier compte de ce maître ait été rendu le 15 novembre $1479^{52}$. Il faut cependant noter que la première mention de la nouvelle date d'entrée en fonction du maître, raturée sur le compte de 1484 , est celle du $1^{\text {er }}$ juin remplacée par le $1^{\text {er }}$ juillet. Le choix de cette date ne correspond pas non plus à une fête importante pour l'institution non plus qu'aux termes des paiements des rentes - Pâques et la Saint-Michel - mais semble cependant choisie pour des facilités de tenue des comptes.

À la fin du siècle, la date de présentation des comptes est à nouveau changée et c'est le terme du 23 juillet qui est choisi sous l'administration de Gautier Chéradames ${ }^{53}$.

Seule la date du compte de 1479 semble faire sens puisque ce compte transitoire est établi à la Saint-Michel, date de perception des rentes et des fermages par l'institution hospitalière.

La clôture d'un compte n'est effective qu'après son audition, laquelle a théoriquement lieu dans un délai restreint. Ainsi le compte de 1402-1403 semble avoir été audité quelques semaines après la fin de l'exercice. Mais il apparait que dans le cas de Charles Lemesnagier, les comptes ne sont audités que de 18 à 23 ans après leur rédaction ${ }^{54}$. Certes l'existence de délais entre les dates de rédaction des comptes et leur reddition est courante au Moyen Âge, comme c'est le cas par exemple pour les comptes de Marie 
d'Anjou, et ce délai tend à s'élargir au fil du temps. Mais aucun des délais attestés par ailleurs n'atteint la durée de ceux constatés à l'hôtel-Dieu argentanais ${ }^{55}$. La période d'audition de l'ensemble des comptes de Charles Lemesnagier, tenus entre 1453 et 1479, s'étend du mois de janvier 1479 au mois de mars de la même année et ces sessions d'audition ont lieu peu avant la mort de ce maître. Ce très long délai d'environ 20 ans peut dans un premier temps s'expliquer par la confiance accordée à ce maitre par les bourgeois d'Argentan, confiance d'autant plus évidente que Lemesnagier est le seul maître désigné « à vie ». L'examen du travail de ce maître, concentré dans le temps, peut aussi être lié à la mort imminente de ce dernier. Les vérifications systématiques de ses registres témoignent alors de la certitude des bourgeois de le voir mourir prochainement et de leur volonté d'apurer les comptes avant sa disparition - et même si sa veuve et ses descendants sont financièrement responsables de sa gestion au même titre que lui. L'examen des dates d'audition montre que celle-ci se fait lors de véritables sessions réunissant deux auditeurs qui siègent entre les mois de janvier et mars 1479 pour entendre les comptes des années 1457-1462 puis à nouveau au mois d'avril 1489 pour recevoir les comptabilités des années 1484-1487.

Le délai entre fin de l'exercice comptable et l'audition tend cependant à diminuer à la fin $\mathrm{du} \mathrm{XV}$ siècle pour se réduire à seulement 5 ans pour les comptes des années 1484-1488.

\section{Les différents types de comptes}

L'existence de différents genres de comptes a déjà pu être notée au sein de l'ensemble comptable hospitalier ${ }^{56}$.

Les comptes définitifs se caractérisent par le respect d'une structure interne immuable, le soin généralement porté à la rédaction et à leur présentation. Tous ont été tenus dans l'optique d'une reddition probable. Cependant, dans ce groupe qui représente la majeure partie des comptes hospitaliers, il est possible de faire une distinction entre les comptes définitifs préparés pour l'audition d'une part - que celle-ci ait été effective ou non - et les comptes n'ayant pas été repris en vue de l'audition ou de leur clôture. Les comptes corrigés pour l'audition se caractérisent tous par l'ajout des sommes de fin de paragraphes et de fin de chapitre, par l'apport de compléments lorsque les comptes ont été pré-rédigés - noms des tenants, sommes perçues voire paragraphes et chapitres entiers - et par l'adjonction de toute information ou complément d'information jugé nécessaire pour l'audition. Cet amendement des comptes avant leur examen entraîne parfois la présence de titres intermédiaires. Tous ces éléments d'information destinés à faciliter la lecture et l'examen des comptes sont aisément repérables dans le manuscrit par la présence au sein du texte d'écritures différentes de celle du scribe ou du maître chargé à l'origine de la rédaction et par des encres distinctes de l'encre utilisée lors de la rédaction initiale.

Les comptes définitifs audités se distinguent par plusieurs types de mentions, d'ajouts ou de corrections portés lors de l'audition à la demande des auditeurs ou du maître. La plupart des éléments écrits au cours de l'audition sont portés dans la marge extérieure de chaque page. Il peut s'agir de ratures du texte original auquel est alors substituée une correction en marge ou au-dessus de l'information raturée; de mentions de délais de paiement ou de justification à l'absence ou au retard d'un paiement; de mentions de pièces justificatives présentées par le maître pendant l'examen de ses comptes. De telles corrections ne sont pas présentes dans tous les comptes audités. Un compte évalué par les 
auditeurs dans la seconde moitié du XV siècle inclut un rappel de la délégation qu'ils ont reçue des bourgeois ${ }^{57}$, le calcul de la totalité des dépenses de l'hôtel-Dieu incluant les frais d'audition ${ }^{58}$ - il s'agit le plus souvent de frais de bouche ${ }^{59}$ - et le paraphe des auditeurs attestant la réception du compte et de sa validité ${ }^{60}$ (fig. 5). Les deux comptes de début de siècle et celui de 1453 diffèrent légèrement puisqu'ils ne portent pas mention de la commission des auditeurs ni trace de leur paraphe. Les dépenses occasionnées par l'audition apparaissent en 1405. Cela démontre une évolution dans les procédures d'audition aussi bien qu'une volonté dans les années 1470 - période d'audition d'un grand nombre de comptes - de vérifier et d'authentifier les comptes pour une meilleure gestion de Saint-Thomas suite aux déboires des années d'occupation et à la longue gestion non contrôlée de Charles Lemesnagier. Le compte de 1486 est le seul à être clôturé par une copie de la commission des auditeurs, acte qui semble d'habitude être un acte notarié annexe conservé le temps de l'audition. Celui de 1487 comprend un acte de réception qui est habituellement induit par le paraphe des auditeurs.

Les comptes définitifs ni audités ni préparés pour l'audition sont repérables par l'absence des mentions et précisions déjà citées. Ceux qui ont été pré-rédigés se remarquent par la multiplicité des mentions incomplètes et des vides émaillant le texte. Dans les comptes non audités, les feuillets destinés à recevoir la somme de la recette et la somme de la dépense sont restés vierges ${ }^{61}$.

41 Il existe en parallèle de cette comptabilité officielle de Saint-Thomas d'autres comptes, évoqués dans les comptes définitifs, dont certains nous sont parvenus. Plusieurs journaux et comptabilités annexes ont été conservés en regard de la comptabilité officielle de l'hôtel-Dieu, ils consistent en journaux annuels utilisés pour rédiger et/ou compléter la comptabilité qui doit être auditée. C'est ce qu'explique très clairement une mention dans le compte de 1458 : «journaux et papiers ordinaires pour faire la recepte et despence dudit compte $\aleph^{62}$. Trois types de documents sont ainsi tenus pour la gestion de l'hôpital : des journaux de la recette des rentes, des journaux des dépenses et un journal du maître.

Les journaux de la recette des rentes portent une liste nominative des tenants de rentes dues à Saint-Thomas, organisée par ordre géographique - en commençant par les paroisses argentanaises puis les paroisses rurales. La liste indique les nom et prénom des payeurs, quelquefois leur profession, le montant de la rente ainsi que le terme de paiement de ces rentes, à savoir la Saint-Michel. Les biens sur lesquels portent ces rentes ne sont ni mentionnés ni détaillés, au contraire du compte définitif. Il est dont probable, et c'est surtout vrai dans les comptes pré-rédigés, que c'est le compte définitif précédent qui sert de référence pour le détail des biens. De plus, les actes de constitution de rente sont précieusement conservés par l'administration hospitalière qui les présente le cas échéant lors de l'audition du compte définitif.

Les journaux des dépenses détaillent quotidiennement chaque type de dépenses, comme par exemple les dépenses de bouche ou encore les frais induits par l'accueil des enfants abandonnés. Il est cependant surprenant qu'à l'instar des comptes définitifs, aucune information sur les malades n'y soit donnée.

44 Aucun journal du maître médiéval n'a été conservé et c'est peut-être à cette comptabilité, probablement tenue au jour le jour et réorganisée en chapitres dans le compte définitif qu'il est fait allusion dans le compte de 1479 sous l'appellation de " comptes ordinaires » du maitre ${ }^{63}$. Ces références laissent entendre que le maître porte dans son journal 
l'ensemble des dépenses qu'il engage, qu'elles relèvent de sa fonction de directeur de l'hôtel-Dieu ou qu'il s'agisse de dépenses privées ${ }^{64}$. Les recettes des aumônes exposées de façon mensuelle dans le compte définitif sont portées de façon hebdomadaire dans le journal du maître ${ }^{65}$.

Si les journaux de la recette des rentes sont tenus en fonction du terme de paiement, la Saint- Michel, le journal du maître suit les termes retenus pour le compte définitif, c'està-dire les dates d'entrée en fonction et de fin de mandat du maitre concerné ${ }^{66}$. Il arrive que l'ensemble de ces actes comptables soient rédigés par le scribe employé à la confection de la comptabilité finale ${ }^{67}$ mais c'est le maître qui le plus souvent en assure la tenue.

Les allusions à ces comptabilités parallèles sont rares dans la comptabilité définitive mais elles permettent de comprendre que toutes les dépenses ou recettes qu'elles comportent ne sont pas systématiquement reportées dans les comptes officiels.

L'absence dans les comptes définitifs de dépenses et de recettes importantes ressort de la lecture de deux actes, un de novembre 1449 et le deuxième de 1495. Le premier est l'acte de désignation de Charles Lemesnagier comme maître et administrateur de l'hôtel-Dieu Saint-Thomas ${ }^{68}$. Il ressort de cet accord que le nouveau maitre doit faire un versement en nature mais également en argent - 200 livres tournois - au profit de l'hôpital. Il est probable que chaque maître nouvellement institué a été soumis à un versement similaire, or ces sommes ne sont jamais exposées dans les comptes définitifs. L'accord de 1495 met fin à l'obligation de l'hôtel-Dieu Saint-Thomas de participer financièrement aux chantiers paroissiaux argentanais. Les sommes sont versées aux fabriques dès que des travaux sont menés à l'une des deux églises paroissiales de la ville - Saint-Martin et Saint-Germain -, ce qui est le cas tout au long du XV siècle. Or, s'il est fait mention d'un versement de ce type en $1495^{69}$, la participation financière de Saint-Thomas aux chantiers paroissiaux n'est habituellement pas inscrite au chapitre des dépenses de la comptabilité hospitalière.

\section{Structure d'un compte définitif}

48 Chaque compte se décompose en quatre parties majeures. Il s'ouvre sur un paragraphe introductif signalant l'identité du maître en place, portant rappel du mandat qu'il détient au nom de la communauté bourgeoise, indiquant les termes d'ouverture et de fin du compte et présentant les deux parties - recettes puis dépenses - et les différents chapitres que le compte comporte.

49 À l'issue de cette présentation, le compte débute par la partie consacrée aux revenus de l'hôtel-Dieu et expose les dépenses dans une seconde partie. En cela, les comtes hospitaliers argentanais suivent la disposition classique des comptes médiévaux scindés en recettes et dépenses. Cette dichotomie recettes/dépenses apparait dès le premier compte de 1402 mais existe déjà dans les comptes du XIV siècle aujourd'hui disparus. L'examen des comptes de Nicole Le Petit en 1374 stipule qu'il a porté sur «la fin de ses comptes tant de recette que de mise $»^{70}$. Suivant ce même ordre, tous les comptes du XV siècle débutent par une présentation des revenus de Saint-Thomas et exposent dans un deuxième temps les dépenses auquel l'hôpital a fait face. Chaque partie du compte est divisée en chapitres secondaires adaptés à la nature des recettes et dépenses de l'hôtelDieu. 
50 La partie relative aux recettes est divisée en trois chapitres majeurs: le revenu du temporel, le revenu des meubles et le revenu des appartenances. Si le chapitre des recettes s'ouvre toujours sur la partie consacrée au revenu du temporel, les deux autres parties n'apparaissent pas toujours dans le même ordre.

51 Le revenu du temporel est lui-même subdivisé en trois sous-parties: rentes, fiefs et domaines muables. Les rentes consistent en l'ensemble des rentes en argent et en nature perçues par l'hôtel-Dieu, présentées dans un ordre géographique immuable, d'abord les rentes en argent sur les paroisses argentanaises puis sur les paroisses rurales puis les rentes en grains dans le même ordre géographique. La partie consacrée aux fiefs vient s'intercaler dans le chapitre des recettes en 1454, date à laquelle Andrieu Noiset, ancien vicomte d'Argentan, est accueilli à Saint-Thomas contre le don de ses deux fiefs de Laillerie et des Champeaux. Le domaine muable regroupe l'ensemble des biens et des droits que l'hôtel-Dieu met à ferme, qu'il s'agisse de biens immeubles ${ }^{71}$, des droits de foire ou de ses droits dans la forêt voisine de Gouffern ${ }^{72}$. Une autre sous-partie vient s'additionner à ces recettes du temporel en 1495. Il s'agit des revenus versés par la confrérie Saint-Nicolas.

Le revenu des meubles énumère l'estimation monétaire des grains et blés des terres de l'hôtel-Dieu, qu'ils soient ou non mis en vente, les bêtes et les charrues de Saint-Thomas et le bénéfice de la vente de meubles. Chacune de ces recettes est détaillée en un paragraphe spécifique. S'y ajoutent parfois des sommes versées pour la réception de nouveaux frères à l'hôtel-Dieu.

53 Le revenu des appartenances est ordonné en cinq sous-parties : le revenu des dîmes de deux paroisses que Saint-Thomas touche depuis sa fondation; le produit de la vente des biens des donnés et malades morts à l'hôtel-Dieu ; la recette des fils de laine et de chanvre produits au bénéfice de Saint-Thomas, qu'ils soient là encore vendus ou non; le produit des quêtes pour le luminaire dans les lieux de culte relevant de l'autorité de SaintThomas ; les dons et l'apport des ostentions de reliques.

Les sommes et grains versés par une centaine de moulins sur lesquels Saint-Thomas touche des "aumônes perpétuelles" ne sont pas toujours indiquées dans le même chapitre. Inscrit avec les revenus du temporel au début du siècle et au début des années 1450, le revenu des moulins est inclus dans le revenu des appartenances à partir du compte de 1457. Aucune information ne vient expliquer ce changement d'affectation alors même que la nature de la recette ne change pas.

Les dépenses se structurent en trois chapitres équivalents aux chapitres de la recette. Les dépenses du temporel énumèrent les frais engagés pour la perception des rentes et des droits divers, pour les messes dites pour les bienfaiteurs et les vivres des frères, les gages et pensions du personnel ainsi que les courtoisies - repas et cadeaux - faites au duc, à ses officiers et à sa maison pour maintenir ces droits. L'acquisition de nouveaux biens est également transcrite dans ce chapitre.

Les dépenses des meubles incluent les frais d'entretien des animaux - fourrage, perte de bétail - et des charrois, les frais de personnel - chambrières et servants divers - et pour finir les dépenses de bouche, d'habillement et de chaussure pour les personnes accueillies, le maître et les condonnés. L'argent dépensé pour le maître et l'ensemble du personnel faisait l'objet d'un chapitre particulier au début $d u X V^{e}$ siècle mais à partir des années 1450, il est inclus de façon systématique dans les dépenses des meubles. 

recettes ou de dépenses pourtant indiquées. Des espaces plus ou moins importants - d'un simple espacement entre deux mots à tout un paragraphe - y sont laissés libres pour accueillir des informations nécessaires mais variables : noms des tenants, montants de dépenses ou de recettes attendues, etc. La solution de la pré-rédaction autorise une belle présentation et un soin dans l'écriture mais ces comptes pré-rédigés n'ont pas toujours été complétés surtout si aucune audition n'en a pas été prévue. Les espaces en ont donc été laissés libres et leur multitude atteste d'une préparation en amont du compte avant son amendement en fin d'exercice comptable.

61 La pré-rédaction des comptes n'est pas la règle et certains textes sont rédigés d'une traite et d'une seule main. Cette technique entraîne un nombre plus important de ratures et de reprise des données et ne permet pas - du fait des délais accordés pour préparer le compte pour l'audition - de soigner la présentation et la calligraphie ${ }^{73}$. 


\section{Les procédures comptables : l'institution et ses comptes}

\section{Les attributions du maître} omniprésent de cette comptabilité. En charge pour un mandat reconductible de trois ans, le maître est désigné oralement par un groupe de bourgeois, les «bourgeois constituants $\gg^{74}$. Cette désignation est ensuite portée par écrit et des copies nous en sont parvenues qui permettent d'établir les obligations et prérogatives du dirigeant de l'hôtelDieu.

63 À la fin du XIV siècle comme au début du siècle suivant, les maîtres sont habituellement des prêtres, parfois curés des deux paroisses argentanaises. La probité morale et la nécessaire conduite des âmes que nécessite toute guérison expliquent pour une large part la désignation d'ecclésiastiques à la tête de la maison-Dieu à ses origines. Mais l'enrichissement de l'hôpital et la complexification des procédures comptables et judiciaires ainsi que les déboires connus pendant l'occupation anglaise incitent les Argentanais à désigner, dans la seconde moitié du $\mathrm{XV}^{\mathrm{e}}$ siècle, des bourgeois au poste envié de maître et administrateur de Saint-Thomas. Le maître, s'il est représentant du groupe bourgeois et membre de celui-ci, n'est jamais cependant un bourgeois particulièrement en vue. Il n'exerce par ailleurs aucune charge et doit se consacrer pleinement à la gestion hospitalière ${ }^{75}$.

64 Les attributions du maître et administrateur sont nombreuses. Il lui revient de gérer l'hôtel-Dieu et ses possessions qu'il peut vendre ou affermer. Il est le seul apte à percevoir les revenus et rentes dont jouit Saint-Thomas dont il est le représentant légal auprès des autorités royales et seigneuriales. À ce titre, il doit faire respecter les droits de l'hôpital y compris devant la justice. Il doit aussi veiller à enrichir le patrimoine hospitalier par des acquisitions - rentes, terres, bâtiments - et maintenir les bâtiments hospitaliers en bon état. Cette gestion est donc celle d'un patrimoine foncier et immobilier considérable. Sa charge prévoit également de recevoir les donnés - personnes qui se donnent avec leurs biens à l'hôpital et y reçoivent le vivre et le couvert jusqu'à leur mort - , veiller à l'accueil des malades, passants et gésineresses. C'est pour justifier de toute cette activité et de sa bonne gestion qu'il se trouve dans l'obligation de rendre des comptes aux bourgeois chaque année.

En 1449, l'opposition des bourgeois et de Thomas Huntyngton, maitre qu'ils avaient pourtant désigné malgré le refus des autorités anglaises, pousse à définir à nouveau les attributions du maître et ses devoirs envers la maison-Dieu. L'acte de désignation de son successeur, signé devant le curé d'Argentan et plusieurs bourgeois délégués, porte donc rappel des obligations du maître et insiste sur l'obligation de reddition de comptes : " lequel Lemesnagier par ce moyen rendra compte bon et loyal et en fourme deue ausdits bourgoys ou a leurs procureurs et commis d'an en an au plus tard ou plaisir et vollenté des dits bourgoys de l'entremise, recepte et despence dudit hospital et deffauz, prouffits, rentes, revenues, aumosnes et appartenances dicellui $»^{76}$. Ce devoir de tenir des comptes selon une forme bien établie se double d'autres conditions imposées au nouveau maître. Il doit maintenir Saint-Thomas en bon état, s'engager à bien l'administrer, donner une partie de ses biens meubles en garantie et si ces clauses n'étaient pas respectées, ses biens et ceux de sa femme seraient 
vendus sans procès ${ }^{77}$. On le voit ici, avant même le souci porté à l'institution et à ses possessions, ce qui préoccupe au premier chef les bourgeois, c'est la tenue de comptes réguliers et leur vérification annuelle.

Le maître est une autorité légitime qui exerce donc une responsabilité morale et financière et le fait d'être redevable de son administration sur ses biens propres et ceux de ses héritiers n'est pas sans conséquence sur la tenue des comptes. Ceux-ci sont donc le reflet de son souci de probité mais aussi de sa personnalité et de sa perception propre de la fonction de maître.

Mais être maître et administrateur de Saint-Thomas, consiste aussi en une fonction honorifique pour le bourgeois qui l'exerce. La fonction pallie l'absence de représentation municipale à Argentan et révèle aux yeux des autorités l'existence d'un groupe bourgeois soudé et distinct du reste de la population par l'exercice de privilèges particuliers. Le maître est un véritable délégué de la bourgeoisie locale et à ce titre il conserve dans un coffre bardé de fer, doté d'une serrure élaborée et installé dans sa chambre, l'ensemble des titres de bourgeoisie. Le maître doit donc être du fait de son rôle au sein de l'hôtelDieu et de son essence - émanation du groupe bourgeois face aux autorités royales et seigneuriales - un homme de confiance, fortuné, possédant une bonne maitrise du calcul, de l'écrit et du droit. Son sens des affaires peut aussi être déterminant dans sa nomination. Il est souvent en contact avec les autorités pour le maintien des droits divers de l'hôtel-Dieu, et certaines qualités sont nécessaires, tel le sens du contact, de la diplomatie mais aussi la fermeté dont il doit faire preuve face à des seigneurs importants, les ducs d'Alençon.

\section{Les différents documents comptables produits ou reçus par le maître / l'organisation de la comptabilité et des archives}

Pour la rédaction des comptes, le maître peut s'appuyer sur un important ensemble de documents qui sont ou non conservés à l'issue de l'audition. Tout d'abord, le maître dispose des différentes comptabilités tenues de façon hebdomadaire ou quotidienne, tel le journal du maître, le journal de la dépense et le journal de la recette des rentes. Ces comptabilités sont connues soit par des références qui y sont faites ponctuellement dans les comptes définitifs, soit par des fragments ou comptes isolés aujourd'hui encore conservés. Les informations portées dans ces trois types de comptabilités diffèrent dans leur forme et dans leur fond de celles inscrites dans les comptes définitifs qui n'en sont pas une simple copie. L'ensemble des journaux de la recette des rentes de Charles Lemesnagier ont été préservés peut-être du fait de sa longue administration et de la vérification tardive de ses comptes en 1479 , qui nécessite une véritable remise à plat des droits et biens hospitaliers ${ }^{78}$. S'ils respectent bien l'ordre géographique de perception des rentes - paroisses argentanaises par quartier puis paroisses rurales - les biens sur lesquels portent les rentes n'y sont pas stipulés, le terme du paiement et l'indication de la perception de la somme due y sont précisés au contraire du compte définitif. Les journaux de recette des rentes sont tenus de façon annuelle, de la saint Michel à la saint Michel suivante - terme de paiement des rentes rurales - sans considération pour les termes de la comptabilité officielle. Aucun journal médiéval du maître, qui porte sur l'ensemble des dépenses faites par le maître en son nom propre comme en son office d'administrateur de Saint-Thomas, n'a été conservé. Ils semblent avoir déjà disparu à l'époque moderne puisqu'il n'en est jamais mention dans l'historiographie locale moderne pourtant riche. 
Ces journaux sont utilisés lors de l'audition pour vérifier si les dépenses portées au compte définitif sont bien les seuls frais relevant de l'hôpital et non du domaine privé du maître ${ }^{79}$. Les journaux du maître sont eux aussi annuels et s'ouvrent en même temps que son compte définitif. Difficile d'affirmer si des cahiers des « dépenses quotidiennes pour aumônes faites aux pauvres et passans ", dont il existe un exemplaire pour le XVI ${ }^{\mathrm{e}}$ siècle ${ }^{80}$ , existent déjà à l'époque médiévale, d'autant plus que de telles dépenses n'apparaissent pas dans les comptes définitifs.

Les mentions diverses d'autres comptabilités notamment dans le compte intermédiaire de 1479 ainsi que l'existence avérée d'autres pièces comptables démontre que la comptabilité présentée aux auditeurs ne représente pas l'ensemble du budget de l'hôtelDieu et que certaines dépenses et recettes sont gérées en parallèle de cette comptabilité « officielle ». Il reste difficile d'établir sur quels critères certaines dépenses et recettes sont ou non intégrées dans la comptabilité définitive et pourquoi.

Des pièces annexes sont nécessaires à la tenue du compte et conservées jusqu'à son audition et sa réception. Pour l'ensemble des rentes et propriétés de l'hôpital, les titres et actes de constitution en sont précieusement archivées par l'administration hospitalière et nombre d'entre eux sont aujourd'hui consultables aux Archives de l'Orne ${ }^{81}$. Les autres pièces - quittances, mandements, cédules obligeant au paiement d'une rente ou d'un droit - ne sont conservées que de façon temporaire et semblent systématiquement éliminées après l'audition ; aucun exemplaire ne nous en est parvenu.

71 La conservation des comptes définitifs ainsi que des pièces comptables majeures - à savoir les autres comptabilités -, la pagination, le soin apporté à la rédaction ainsi que l'emploi de supports de qualité démontrent une volonté précoce de conservation et d'utilisation à long terme des comptes et un souci archivistique ancien. Pour les conserver, le dépôt dans des coffres ou des armoires est attesté avec les pièces d'écriture les plus précieuses à savoir les titres de propriété, les fondations et les donations de droits ou de terre.

\section{La procédure d'audition}

L'audition n'est donc pas seulement un acte oral, elle s'appuie pour une large part sur des documents écrits, présentés comme justificatifs et qui ne sont apparemment pas conservés après réception du compte ${ }^{82}$. Le compte transitoire de 1479 témoigne de cette pratique de présentation d'écrits lors de l'audition et détaille ces titres et actes montrés pour justifier la bonne tenue des comptes par le maître. Il s'agit pour l'essentiel d'actes notariés - actes de constitution de rente, cédules, comptes - permettant d'établir l'origine d'une rente et/ou son bien-fondé, de justifier la perception de droits ou de rentes. La nature de ces documents n'est pas toujours spécifiée mais leur présentation lors de l'audition fait en 1479 l'objet d'une note marginale systématique, écrite en regard du texte comptable. Dans cette période de transition et de changement de maitre à la tête de l'hôtel-Dieu, il s'agit d'établir le bon droit de l'hôpital, la légitimité de ses ressources et l'exactitude de ses dépenses afin d'éviter toute contestation. Certains des textes produits alors datent du début du XIII ${ }^{e}$ siècle, ce qui prouve le sérieux de l'audition aussi bien que l'existence d'archives organisées de longue date à l'hôtel-Dieu. L'absence de justificatifs écrits est elle aussi stipulée lors de cette audition.

La fiabilité des actes présentés peut par ailleurs, en plus de leur présentation aux auditeurs, faire l'objet d'une authentification attestée par l'ajout d'une note marginale, 
comme par exemple ce commentaire établissant la véracité d'une rente versée par un nommé Noël Chestien : « le texte est véritable »"

Mais l'audition du compte même dans le cas exceptionnel de 1479 reste avant tout un acte oral et c'est alors la parole du maître qui est entendue. Ces indications peuvent lui être demandées pendant l'audition, elles sont elles aussi notifiées dans la marge extérieure. Les termes usités dans les notes marginales attestent de cette pratique, « le maistre dit que lesdits héritages sont baillés à rentes ", ou encore « ledit maistre tesmoigne avoir veu le compte $»^{84}$. Pour attester sa bonne foi et son bon droit, le maître peut également jurer : « ledit maistre a fait foy qu'il n'en a rien reçeu $»^{85}$. Les attestations et précisions verbales qu'il apporte ne sont pas dénuées de solennité puisqu'il en "fait foi» ou qu'il en « témoigne » ou qu'il « jure » selon les termes employés dans ce compte.

L'audition ne se termine pas avec la présentation d'actes ou l'apport d'informations orales par le maître. Les auditeurs peuvent exiger de lui qu'il s'engage à fournir des précisions ultérieures - s'il n'est pas en mesure de se justifier immédiatement - ou à percevoir les sommes dues dans les meilleurs délais, à charge pour lui d'en présenter justification dans ses journaux de la recette. Il s'agit ici de la part des auditeurs de véritables injonctions à faire son devoir de responsable du bien commun ${ }^{86}$. Les auditeurs peuvent pareillement lui enjoindre de contraindre les débiteurs de l'hôpital de s'acquitter de leurs dettes voire de recourir à la justice pour obtenir le paiement de rentes non perçues $^{87}$ (tableau 5).

\section{Évolution constatée}

76 La densité d'informations transcrites dans les comptes est à la fois variable selon le maître et la période. Des périodes de reprise en main de la gestion de la comptabilité et du patrimoine hospitaliers alternent avec des périodes de relâchement très visibles dans la tenue des comptes par l'écriture, le soin apporté à la rédaction et le détail et la précision des recettes et des dépenses. Ces périodes pendant lesquelles les comptes sont moins bien tenus sont à lier avec les difficultés de gestion de l'hôtel-Dieu pendant l'occupation anglaise pour laquelle aucun compte n'a été rédigé et/ou ne nous est parvenu mais aussi à une véritable usure du pouvoir, notamment sensible dans les comptes de fin d'administration de Charles Lemesnagier, en poste pendant une trentaine d'années. La confiance dont il jouit auprès des bourgeois - il est le seul maître nommé à vie -, la compétence dont il a fait preuve pour rétablir les finances et les droits de l'institution et la durée exceptionnelle de son mandat justifient cet apparent laisser-aller.

77 Les effets de la guerre et de l'occupation se font sentir et les comptes des années 1450 sont particulièrement précis; ils témoignent de la réforme de l'administration hospitalière et des procédures comptables. Une reprise en main de l'hôpital et de sa gestion se perçoit donc nettement dans les premiers comptes d'après-guerre qui font le bilan des droits et biens de Saint-Thomas et les détaillent en en indiquant l'origine (date, nom des tenants, etc.). Les comptes sont alors tenus avec rigueur et précision. C'est aussi le cas du compte de 1479 établi à la mort d'un maitre longtemps en place, Charles Lemesnagier. Il convient alors de faire le point sur l'état financier et mobilier de l'hôpital mais aussi de retrouver l'origine de biens et de dons certes connus du précédent maître mais qui risquent alors d'être contestés par les tenants. L'organisation de la comptabilité hospitalière se fait dès le $\mathrm{XV}^{\mathrm{e}}$ siècle, dans le but de clarifier les trente années d'administration de Charles Lemesnagier. Ses comptes sont alors numérotés dans l'ordre 
chronologique. Cette numérotation est faite en chiffres romains en haut de la première page de chaque compte et spécifie qu'il s'agit d'un compte tenu par Charles Lemesnagier.

Les comptes de la fin du siècle sont plus lapidaires. L'hôtel-Dieu ayant alors réorganisé ses archives et rétabli ses droits et propriétés, il ne semble plus dès lors nécessaire de détailler chaque rente et son origine ni de faire rappel des actes de fondation.

Dès le $\mathrm{XVI}^{\mathrm{e}}$ siècle, les registres comptables médiévaux sont utilisés pour établir des inventaires des titres et revenus de l'hôtel-Dieu. Ainsi, l'inventaire de 1555 s'inspire probablement de ces comptabilités dont il suit l'ordre des chapitres ${ }^{88}$. Les comptes sont donc à la fois objets de conservation et outils de référence pour le classement moderne des autres titres de Saint-Thomas. Ils servent aussi de preuve en cas de litige pour la perception de droits ou la propriété de biens.

\section{La valeur de la source}

\section{Valeur pour l'étude des comptabilités médiévales}

80 Les comptes médiévaux de Saint-Thomas offrent un exemple très intéressant de comptabilité du XVe siècle. Cette longue série, bien que majoritairement de la seconde moitié du XVe siècle, permet de percevoir l'évolution des techniques comptables entre le début et la fin du siècle. Elle autorise aussi à établir les permanences et habitudes des gestionnaires de l'hôtel-Dieu et celles des scribes chargés d'en assurer la rédaction. Le compte de transition de 1479 est plus particulièrement intéressant par les nombreuses indications qu'il livre sur la préparation et la construction des comptes et sur les procédures d'audition qu'il détaille avec beaucoup de soin. Au travers de cet ensemble comptable c'est aussi l'importance du maître dans l'élaboration et la présentation du compte qui est perceptible. Les comptes transitoires et les comptes de début de mandat fourmillent de précisions qui tendent à disparaître lorsque le maître reste longtemps en charge et que l'exercice comptable lui devient familier. Le contrôle exercé par les représentants des bourgeois sur l'institution hospitalière et la vérification qu'ils mènent sur les comptes semblent également avoir un fort impact sur leur tenue. Si tous les comptes sont lisibles, respectent certaines normes de présentation et se veulent un reflet clair de la gestion du maître, c'est que la vérification par les auditeurs en est précise et détaillée.

\section{Valeur indirecte}

Dès le XIX ${ }^{\mathrm{e}}$ siècle, l'importance de cette source pour l'histoire de la ville d'Argentan a été bien connue, comme en témoignent les nombreux passages soulignés dans les comptes, les commentaires qui y ont été écrits en marge mais aussi la constitution d'extraits de comptes aujourd'hui intégrés dans la série $\mathrm{H}$.

82 C'est avant tout l'étude de l'institution hospitalière qui a été construite sur la base des comptes hospitaliers médiévaux. Plusieurs articles ont été basés sur les comptes hospitaliers argentanais, soit dans l'optique de l'alimentation soit dans celle de la population accueillie par l'hôpital. Mais la comptabilité de Saint-Thomas se révèle également une source sans égale pour l'étude de la topographie et de la toponymie argentanaises, pour la connaissance des pouvoirs en place - et plus particulièrement de la relation du groupe bourgeois à la famille ducale d'Alençon - et pour une approche du 
tissu artisanal, commercial et économique de la ville. Cette comptabilité n'est d'ailleurs pas exclusivement urbaine et réserve de nombreux paragraphes aux paroisses rurales du plat-pays, permettant ainsi de percevoir les relations de la ville à sa proche campagne, les liens économiques et politiques qui les lient et la volonté urbaine de contrôler les ressources campagnardes et d'affirmer son autorité.

Datée de la seconde moitié du $\mathrm{XV}^{\mathrm{e}}$ siècle, la comptabilité se révèle une source indispensable pour connaitre l'impact de la guerre de cent ans et de l'occupation anglaise sur la région. L'étendue des destructions qu'elle évoque, les rentes que l'hôtel-Dieu ne perçoit plus dans les années 1450 démontrent l'importance des dégâts occasionnés par la guerre et les troubles qui la suivent ainsi que la destructuration de l'économie locale. En faisant mention des habitants qui peuplent la ville, les registres ne peuvent ignorer l'installation d'Anglais dans ses murs, qu'ils soient soldats ou membres de l'administration lancastrienne. Cette présence anglaise est nettement perceptible dans les comptes par les rentes versées par l'occupant, l'implication des autorités anglaises dans la gestion de l'hôtel-Dieu et dans celle de la ville mais aussi par ce qu'ils donnent à voir des relations entre habitants et Anglais. Les comptes démontrent aussi l'implication de bourgeois dans l'administration d'occupation et la continuité de l'administration seigneuriale, bourgeoise et hospitalière avant, pendant et après l'occupation.

Cependant, malgré l'apport indiscutable qu'elle constitue, la comptabilité hospitalière ne peut être conçue comme une source isolée et la lecture doit s'en faire en parallèle de l'autre comptabilité urbaine conservée - les comptes de fabrique de Saint-Germain d'Argentan - et des comptabilités rurales proches - comptes de la vicomté d'Exmes et comptes de l'abbaye prémontrée de Silly - qui viennent la compléter et en permettre la critique.

\section{BIBLIOGRAPHIE}

Bos, Agnès, « Les archives des fabriques parisiennes à la fin du Moyen Âge et à l'époque moderne ", Bibliothèque de l'École des chartes, tome 156, juillet-décembre 1998, p. 369-405.

BRÉARD, Charles, «Le compte du clos des galées de Rouen pour 1382-1384 », in Mélanges, Ch. DE BEAUREPAIRE, l'abbé BLANQUART, Ch. BRÉARD et al. (éd.), Rouen, Lestringant, 1893, 2e série, p. 53-154.

BRIQUET, Charles-Moïse, Les filigranes. Dictionnaire historique des marques du papier dès leur apparition vers 1282 jusqu'en 1600, fac-similé de l'édition de 1923, Martino Publishing, Mansfield, 2000, 4 tomes.

COURTEILLES, Michel (de), Description sincère et fidelle de la ville d'Argentan curieusement recherchée en 1693 et 1694 par un prêtre anonyme, Médiathèque d'Argentan, ms 63, 646 p., 28 ill.

CUVEILLER, Stéphane, Dunkerque, ville et port de Flandre à la fin du Moyen Âge à travers les comptes de bailliage de 1387 à 1407, Lille, Presses universitaires de Lille, 1989, 374 p.

DÉSERT, Gabriel, Les archives hospitalières : source d'histoire économique et sociale, Caen, Annales de Normandie (coll. des Cahiers des Annales de Normandie, $\left.\mathrm{n}^{\circ} 10\right), 1977,250 \mathrm{p}$. 
DUBoIs, Jacques, Notre-Dame d'Alençon. Financement et reconstruction (1350-1540), Rennes, Presses universtaires de Rennes, 2000, $194 \mathrm{p}$.

DUBoIs, Mariannick, « L'hôtel-Dieu d'Argentan au Moyen Âge (XIII ${ }^{\mathrm{e}}-\mathrm{XV}^{\mathrm{e}}$ siècle) », Bulletin de la Société française d'histoire des hôpitaux, n 34, 1977, p. 46-48.

FAZY, Max, Notes et documents sur l'hôtel-Dieu d'Alençon, Alençon, Impr. Herpin, 1913, 201 p.

GIBELLO, Corinne, « L'hôtel-Dieu Saint-Thomas d'Argentan des origines à la veille de la

Révolution ", Bulletin de la Société historique et archéologique de l'Orne, tome CVII, $\mathrm{n}^{\circ} 4$, décembre 1988, p. 29-31.

GOLDMAN, Catherine, Les Garencières-le-Baveux, vie et mort d'un lignage de la moyenne noblesse normande (fin $\mathrm{XIII}^{e}-\mathrm{XV}{ }^{e}$ siècle), Livre III : Le compte de la seigneurie de Fontenay-le-Marmion, thèse de l'université de Paris IV - Sorbonne, 1991, 133 p. (dactyl.).

GREVE, Anke et LEBAILly, Émilie (éd.), Comptes de l'argentier de Charles le Téméraire, duc de Bourgogne, Paris, de Boccard, 2001-2002, 2 vol., 612 et 549 p.

IMBERT, Jean (dir.), Histoire des hôpitaux en France, Toulouse, Privat, 1982, 559 p.

LAURENT, Eugène (abbé), Saint-Germain d'Argentan, diocèse de Séez : Histoire d'une paroisse catholique pendant les trois derniers siècles, Argentan, Impr. Barbier, 1859, 573 p.

LE CACHEUX, Paul, Essai historique sur l'hôtel-Dieu de Coutances, l'hôpital général et les Augustines hospitalières depuis l'origine jusqu'à la Révolution avec cartulaire général, tome 1, L'hôtelDieu 1209-1789, Paris, Picard et fils, 1895, 340 p.

LOUIS, Ulysse, РАJOT, Pierre et POUËSSEL, Jean, Le livre de rentes de l'hôtel-Dieu de Vernon, Saint-Aubinles-Elbeuf, éd. Page de garde, 1997, 132 p.

Mollat, Michel, Comptabilité du port de Dieppe au XVe siècle , Paris, Colin, 1951, 138 p.

MOULIN, Marie-Anne, Argentan à la fin du Moyen Âge : aspects urbains, sociaux et économiques, thèse de l'EHESS sous la direction de P. BRAUNSTEIN, 2007, 4 vol. texte + 1 vol. ill., 940 p. (dactyl.).

NEVEUX, François, «Les établissements d'assistance », in La Normandie royale (XIII ${ }^{e}-X^{2} V^{e}$ s.), François NEVEUX (éd.), Rennes, éd. Ouest France Université, 2005, p. 217-241.

NEVEUX, François, La Normandie royale (XIII ${ }^{e}-X V^{e}$ s.), Rennes, éd. Ouest France Université, 2005, $556 \mathrm{p}$.

SAUNIER, Annie, « La clientèle de Saint-Thomas d'Argentan entre 1450 et 1521 : malades, gésineresses et jettés ", in Marguerite de Lorraine et son temps, 1463-1521, bulletin spécial de la Société historique et archéologique de l'Orne, 1989, p. 133-159.

VÉRITÉ, Isabelle, « Les comptes des trésoriers de l'église Saint-Germain d'Argentan, 1410-1476 : la gestion d'une église au XVI ${ }^{\mathrm{e}}$ siècle », thèse de IIIe cycle, Université de Paris X, 1990.

VÉRITÉ, Isabelle, « La gestion du trésor de Saint-Germain d'Argentan (diocèse de Sées).

Permanences et évolutions au XV siècle ", in Recherches sur l'économie ecclésiale à la fin du Moyen Âg : autour des collégiales de Savoie, Actes de la table ronde d'Annecy, 26-28 avril 1990, Académie Salésienne, Mémoires et documents n 97, 1991, p. 151-167.

VÉRITÉ, Isabelle, « Les trésoriers de Saint-Germain d'Argentan au XV $\mathrm{XV}^{\mathrm{e}}$ siècle, des notables au service de la ville et de l'Église ", in Argentan et ses environs au Moyen Âge, approche historique et archéologique, Marie-Anne MOULIN, Isabelle CHAVE, Bruno FAJAL et Jean-Pascal FOUCHER (dir.), Alençon, Conseil général de l'Orne, 2008, p. 207-230. 


\section{ANNEXES}

Tableau $\mathrm{n}^{\circ} 1$ : Tableau des comptes

\begin{tabular}{|c|c|c|c|c|}
\hline $\begin{array}{l}\text { Période couverte } \\
\text { (maître en charge) }\end{array}$ & $\begin{array}{l}\text { État du compte } \\
\text { (chapitres et } \\
\text { folios manquants) }\end{array}$ & $\begin{array}{l}\text { Nature } \quad d u \\
\text { compte }\end{array}$ & $\begin{array}{l}\text { Préparation } \\
\text { pour l'audition }\end{array}$ & $\begin{array}{l}\text { Date } \\
\text { d'audition }\end{array}$ \\
\hline $\begin{array}{l}1402-1403 \\
\text { (Colin Guérié) }\end{array}$ & $\begin{array}{l}\text { Incomplet } \\
-\quad \text { chapitre des } \\
\text { dépenses } \\
\text { incomplet }\end{array}$ & $\begin{array}{l}\text { Compte } \\
\text { définitif pré- } \\
\text { rédigé }\end{array}$ & Oui & inconnue \\
\hline $\begin{array}{l}\text { 1405-1406 } \\
\text { (Pierre Doulxfrère) }\end{array}$ & $\begin{array}{l}\text { Incomplet } \\
\text { - premières pages } \\
\text { manquantes }\end{array}$ & $\begin{array}{l}\text { Compte } \\
\text { définitif }\end{array}$ & & inconnue \\
\hline $\begin{array}{l}\text { 1431-1433 } \\
\text { (Robert Le Rouxelet) }\end{array}$ & Incomplet & $\begin{array}{ll}\text { Journal } & \text { du } \\
\text { maître } & \text { ou } \\
\text { journal } & \text { des } \\
\text { recettes } & \text { des } \\
\text { rentes }^{89} & \end{array}$ & $\begin{array}{l}\text { Comptabilité à } \\
\text { usage interne, } \\
\text { non auditée }\end{array}$ & Non \\
\hline $\begin{array}{l}\text { 1453-1454 } \\
\text { (Charles Lemesnagier) }\end{array}$ & $\begin{array}{l}\text { Incomplet } \\
\text { - fin du compte } \\
\text { manquante }\end{array}$ & $\begin{array}{l}\text { Compte } \\
\text { définitif }\end{array}$ & Oui & $\begin{array}{l}22 / 01 / 1477 \\
\text { (n. st.) }\end{array}$ \\
\hline $\begin{array}{l}\text { 1454-1455 } \\
\text { (Charles Lemesnagier) }\end{array}$ & Complet & $\begin{array}{l}\text { Compte } \\
\text { définitif }\end{array}$ & Oui & 01/1477 (n. st.) \\
\hline $\begin{array}{l}1456-1457 \\
\text { (Charles Lemesnagier) }\end{array}$ & $\begin{array}{l}\text { Incomplet } \\
\text { - le compte s'arrête } \\
\text { après le chapitre } \\
\text { des dépenses de } \\
\text { bouche }\end{array}$ & $\begin{array}{l}\text { Compte } \\
\text { définitif }\end{array}$ & Non & Non \\
\hline $\begin{array}{l}1457-1458 \\
\text { (Charles Lemesnagier) }\end{array}$ & Incomplet? & $\begin{array}{l}\text { Compte } \\
\text { définitif }\end{array}$ & Oui & 03/1479 (n. st.) \\
\hline $\begin{array}{l}\text { 1458-1459 } \\
\text { (Charles Lemesnagier) }\end{array}$ & Incomplet? & $\begin{array}{l}\text { Compte } \\
\text { définitif }\end{array}$ & Oui & 03/1479 (n. st.) \\
\hline $\begin{array}{l}\text { 1460-1461 } \\
\text { (Charles Lemesnagier) }\end{array}$ & Complet & $\begin{array}{l}\text { Compte } \\
\text { définitif }\end{array}$ & Oui & 03/1479 (n. st.) \\
\hline $\begin{array}{l}\text { 1461-1462 } \\
\text { (Charles Lemesnagier) }\end{array}$ & \begin{tabular}{|l} 
Incomplet \\
- pas de chapitre \\
des rentes non \\
perçues
\end{tabular} & $\begin{array}{l}\text { Compte } \\
\text { définitif }\end{array}$ & Oui & Inconnue \\
\hline
\end{tabular}




\begin{tabular}{|c|c|c|c|c|}
\hline $\begin{array}{l}1467-1468 \\
\text { (Charles Lemesnagier) }\end{array}$ & $\begin{array}{l}\text { Incomplet } \\
\text { - chapitre des } \\
\text { dépenses de } \\
\text { bouche incomplet } \\
\text { - fin du compte } \\
\text { manquante }\end{array}$ & $\begin{array}{l}\text { Compte } \\
\text { définitif }\end{array}$ & Oui & Pas d'audition \\
\hline $\begin{array}{l}1468-1469 \\
\text { (Charles Lemesnagier) }\end{array}$ & \begin{tabular}{|lr} 
Incomplet & \\
- pas de chapitre \\
des rentes non \\
perçues
\end{tabular} & $\begin{array}{l}\text { Compte } \\
\text { définitif pré- } \\
\text { rédigé }\end{array}$ & Non & Pas d'audition \\
\hline $\begin{array}{l}1470-1471 \\
\text { (Charles Lemesnagier) }\end{array}$ & $\begin{array}{l}\text { Incomplet } \\
\text { - fin du chapitre } \\
\text { des rentes non } \\
\text { perçues manquant }\end{array}$ & $\begin{array}{l}\text { Compte } \\
\text { définitif }\end{array}$ & Non & Pas d'audition \\
\hline $\begin{array}{l}\text { 1473-1474 } \\
\text { (Charles Lemesnagier) }\end{array}$ & $\begin{array}{l}\text { Incomplet } \\
\text { - fin du compte } \\
\text { endommagée } \\
\text { - chapitre des } \\
\text { dépenses } \\
\text { incomplet }\end{array}$ & $\begin{array}{l}\text { Compte } \\
\text { définitif }\end{array}$ & Non & Pas d'audition \\
\hline $\begin{array}{l}1474-1475 \\
\text { (Charles Lemesnagier) }\end{array}$ & Complet? & $\begin{array}{l}\text { Compte } \\
\text { définitif }\end{array}$ & Oui & Pas d'audition \\
\hline $\begin{array}{l}1475-1476 \\
\text { (Charles Lemesnagier) }\end{array}$ & $\begin{array}{l}\text { Complet } \\
\text { - quelques pages } \\
\text { endommagées }\end{array}$ & $\begin{array}{l}\text { Compte } \\
\text { définitif }\end{array}$ & Non & Pas d'audition \\
\hline Saint-Michel 1479 & Complet & $\begin{array}{l}\text { Compte de } \\
\text { transition }\end{array}$ & Oui & Inconnue \\
\hline $\begin{array}{l}\text { 1484-1485 } \\
\text { (Macé Gaultier) }\end{array}$ & Complet & $\begin{array}{l}\text { Compte } \\
\text { définitif }\end{array}$ & Oui & 04/1489 (n. st.) \\
\hline $\begin{array}{l}\text { 1486-1487 } \\
\text { (Macé Gaultier) }\end{array}$ & Complet & $\begin{array}{l}\text { Compte } \\
\text { définitif }\end{array}$ & Oui & 04/1489 (n. st.) \\
\hline $\begin{array}{l}\text { 1487-1488 } \\
\text { (Macé Gaultier) }\end{array}$ & Complet & $\begin{array}{l}\text { Compte } \\
\text { définitif }\end{array}$ & Oui & $\begin{array}{l}\text { Inconnue } \\
\text { - compte reçu le } \\
19 / 02 / 1493 \\
\text { après } \\
\text { contestation }\end{array}$ \\
\hline $\begin{array}{l}\text { 1488-1489 } \\
\text { (Macé Gaultier) }\end{array}$ & Complet & $\begin{array}{l}\text { Compte } \\
\text { définitif }\end{array}$ & Oui & Pas d'audition \\
\hline $\begin{array}{l}\text { 1489-1490 } \\
\text { (Macé Gaultier) }\end{array}$ & $\begin{array}{l}\text { Incomplet } \\
\text { - pas de chapitre } \\
\text { des dépenses }\end{array}$ & $\begin{array}{l}\text { Compte } \\
\text { définitif }\end{array}$ & Oui & ? \\
\hline $\begin{array}{l}\text { 1491-1492 } \\
\text { (Macé Gaultier) }\end{array}$ & $\begin{array}{l}\text { Incomplet } \\
\text { - deux chapitres: } \\
\text { rentes dans et hors } \\
\text { la ville }\end{array}$ & $\begin{array}{l}\text { Compte } \\
\text { définitif }\end{array}$ & Oui & ? \\
\hline
\end{tabular}




\begin{tabular}{|c|c|c|c|c|}
\hline $\begin{array}{l}\text { 1495-1496 } \\
\text { (Gaultier Chéradames) }\end{array}$ & Complet & $\begin{array}{l}\text { Compte } \\
\text { définitif }\end{array}$ & Oui & Non \\
\hline $\begin{array}{l}\text { 1496-1497 } \\
\text { (Gaultier Chéradames) }\end{array}$ & Complet & $\begin{array}{l}\text { Compte } \\
\text { définitif }\end{array}$ & Partiellement & Non \\
\hline $\begin{array}{l}\text { 1497-1498 } \\
\text { (Gaultier Chéradames) }\end{array}$ & Complet & & & \\
\hline $\begin{array}{l}\text { 1498-1499 } \\
\text { (Gaultier Chéradames) }\end{array}$ & $\begin{array}{l}\text { Incomplet } \\
\text { - pas de chapitre } \\
\text { des rentes non } \\
\text { perçues }\end{array}$ & & & \\
\hline
\end{tabular}

Tableau $n^{\circ} 2$ : Supports et dimensions des cahiers inclus dans les registres hospitaliers

\begin{tabular}{|l|l|l|l|}
\hline Comptes & Support & Nombre de cahiers & Dimensions \\
\hline $1402-1403$ & parchemin & 2 cahiers & $265 \mathrm{~mm} \times 315$ \\
\hline $1405-1406$ & parchemin & 6 cahiers & $280 \mathrm{~mm} \times 350$ \\
\hline $1431-1433$ & papier & 1 cahier & $210 \mathrm{~mm} \times 300$ \\
\hline $1453-1454$ & parchemin & 6 cahiers & $275 \mathrm{~mm} \times 334$ \\
\hline $1454-1499$ & papier & De 2 à 5 cahiers & $220 \mathrm{~mm} \times 300$ \\
\hline
\end{tabular}

Tableau $n^{\circ} 3$ : Rédacteurs, rédaction et ajouts médiévaux dans les comptes hospitaliers

\begin{tabular}{|c|c|c|c|c|c|}
\hline Compte & Rédacteur & $\begin{array}{l}\text { Compte } \\
\text { pré- } \\
\text { rédigé }\end{array}$ & $\begin{array}{l}\text { Nombre de mains } \\
\text { (dans le corps du } \\
\text { texte) }\end{array}$ & $\begin{array}{l}\text { Soin apporté à la } \\
\text { rédaction }\end{array}$ & \begin{tabular}{|l} 
Notes \\
marginales \\
(nature
\end{tabular} \\
\hline $1402-1403$ & Maître? & Oui & 1 main & $\begin{array}{l}\text { Présentation très } \\
\text { soignée; } \\
\text { - majuscules } \\
\text { travaillées. }\end{array}$ & $\begin{array}{l}2 \text { mains (maître } \\
\text { ou scribe); } \\
\text { - précisions }{ }^{90} \text {; } \\
\text { - des ajouts et } \\
\text { corrections lors } \\
\text { de l'audition. }\end{array}$ \\
\hline $1405-1406$ & scribe & Non & 1 main & $\begin{array}{l}\text { Compte très soigné } \\
\text { - Lettrines initiales } \\
\text { décorées (figure du } \\
\text { Christ p.61, profil de } \\
\text { moine p.63, figure } \\
\text { d'homme p. } 65, \\
\text { initiale fleurie p. } 73, \\
\text { tête couronnée, } \\
\text { p. 101). }\end{array}$ & $\begin{array}{ll}2 \text { mains } & \\
- \text { sommes }^{91} & \\
\text { (auditeurs); } & \\
\text { - corrections } & \\
\text { (maître ou } \\
\text { scribe) }\end{array}$ \\
\hline
\end{tabular}




\begin{tabular}{|c|c|c|c|c|c|}
\hline $1453-1454$ & Maître? & Non & 1 main & $\begin{array}{l}\text { - compte soigné ; } \\
\text { - quelques belles } \\
\text { initiales. }\end{array}$ & $\begin{array}{l}2 \text { mains } \\
\text { - sommes } \\
\text { (auditeurs); } \\
\text { - précisions, } \\
\text { corrections, } \\
\text { ratures }^{92} \text { (maître } \\
\text { ou scribe). }\end{array}$ \\
\hline $1454-1455$ & Maître? & Non & $\begin{array}{l}2 \text { mains : } \\
-1 \\
\text { l'intégralité pour } \\
\text { texte; } \\
-1 \text { pour la fin des } \\
\text { rentes en non } \\
\text { valoir (p. 285-287). }\end{array}$ & $\begin{array}{l}\text { - très belles initiales } \\
\text { (fig. 1); } \\
\text { - titres de chapitres } \\
\text { et de paragraphes } \\
\text { soignés. }\end{array}$ & $\begin{array}{l}2 \text { mains (fig. 5) } \\
\text { - sommes de fin } \\
\text { de chapitre } \\
\text { (auditeurs); } \\
\text { - frais d'audition } \\
\text { (maître ou } \\
\text { scribe). }\end{array}$ \\
\hline $1456-1457$ & $\begin{array}{l}\text { Scribe et } \\
\text { maître? }\end{array}$ & Oui & $\begin{array}{l}2 \text { mains : } \\
\text { - texte; } \\
\text { - rajouts de noms } \\
\text { de personnes et de } \\
\text { quartiers. }\end{array}$ & \begin{tabular}{ll} 
Compte & mal \\
présenté, & très \\
incomplet, & mal \\
organisé ; & \\
Écriture peu soignée ; & \multicolumn{2}{l}{ Aucune lettrine. }
\end{tabular} & $\begin{array}{l}2 \text { mains } \\
\text { sommes } \\
\text { (auditeurs); } \\
\text { - corrections, } \\
\text { paragraphes } \\
\text { barrés, ratures } \\
\text { (maître ou } \\
\text { scribe). }\end{array}$ \\
\hline $1457-1458$ & 3 scribes & Non & $\begin{array}{l}3 \text { mains : } \\
\text { - Romain Pigache } 1 \\
\text { ère moitié du } \\
\text { compte; } \\
\text { - changement de } \\
\text { main à partir du } \\
\text { paragraphe sur les } \\
\text { achats de liens; } \\
\text { - confrérie Saint- } \\
\text { Nicolas. }\end{array}$ & Compte peu soigné & $\begin{array}{ll}1 \text { main } & \\
\text { - précisions } & \\
\text { (maître } & \text { ou } \\
\text { scribe); } & \\
\text { - sommes } & \\
\text { (auditeurs); } & \\
\text { - paragraphe } & \text { de } \\
\text { réception } & \text { du } \\
\text { compte } & \\
\text { (auditeurs ?). }\end{array}$ \\
\hline $1458-1459$ & 2 scribes & Oui & $\begin{array}{l}2 \text { mains : } \\
\text { - } 2 \text { mains sur la } 1^{\text {ère }} \\
\text { page ; } \\
\text { - puis R. Pigache. }\end{array}$ & Compte assez soigné & $\begin{array}{l}3 \text { mains : } \\
\text { - précisions } \\
\text { (maître ou } \\
\text { scribe); } \\
\text { - sommes de fin } \\
\text { de paragraphes } \\
\text { (auditeurs); } \\
\text { - sommes de fin } \\
\text { de recette } \\
\text { (auditeurs). }\end{array}$ \\
\hline $1460-1461$ & scribe & Non & $\begin{array}{l}2 \text { mains : } \\
\text { - } 1 \text { main } 1^{\text {ère }} \text { page } \\
\text { - puis R. Pigache }\end{array}$ & $\begin{array}{l}\text { Titres assez soignés } \\
\text { Pas de lettrines } \\
\text { Peu de ratures }\end{array}$ & $\begin{array}{l}2 \text { mains } \\
\text { (auditeurs): } \\
\text { - sommes de fin } \\
\text { de paragraphes } \\
\text { - sommes de fin } \\
\text { de recette }\end{array}$ \\
\hline
\end{tabular}




\begin{tabular}{|c|c|c|c|c|c|}
\hline 1461-1462 & scribe & Non & Romain Pigache & $\begin{array}{l}\text { Titres assez soignés } \\
\text { Pas de lettrines } \\
\text { Peu de ratures }\end{array}$ & $\begin{array}{l}3 \text { mains : } \\
\text { - précisions } \\
\text { (maître ou } \\
\text { scribe); } \\
\text { - sommes de fin } \\
\text { de paragraphes } \\
\text { (auditeurs); } \\
\text { - sommes de fin } \\
\text { de recette } \\
\text { (auditeurs). }\end{array}$ \\
\hline $1467-1468$ & Maître? & Non & 1 main & Compte assez soigné & aucune \\
\hline $1468-1469$ & Maître? & Oui & 1 main & Soin de la rédaction & aucune \\
\hline $1470-1471$ & Maître? & Non & Main $1468^{93}$ & $\begin{array}{l}\text { Soin de la rédaction } \\
\text { (fig. 2) }\end{array}$ & $\begin{array}{l}1 \text { main : } \\
\text { - précisions et } \\
\text { rajouts (maître } \\
\text { ou scribe) }\end{array}$ \\
\hline $1473-1474$ & Maître? & Non & Main 1468 & Soin de la rédaction & aucune \\
\hline 1474-1475 & Maître? & Non & Main 1468 & 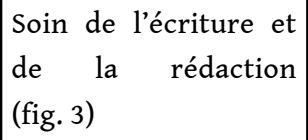 & aucune \\
\hline $1475-1476$ & Maître? & Non & Main 1468 & $\begin{array}{l}\text { Compte beaucoup } \\
\text { plus détaillé que les } \\
\text { précédents, peu de } \\
\text { ratures }\end{array}$ & $\begin{array}{l}1 \text { main (scribe } \\
\text { ou maitre) : } \\
\text { paragraphe des } \\
\text { foires } \\
\text { (p. 215-216) }\end{array}$ \\
\hline 1479 & Scribe & Non & 1 main & $\begin{array}{l}\text { Très belle } \\
\text { présentation: } \\
\text { lettrines et capitales } \\
\text { très soignées }\end{array}$ & $\begin{array}{l}2 \text { mains } \\
\text { (auditeurs): } \\
\text { - sommes; } \\
\text { - précisions en } \\
\text { marge quant à la } \\
\text { procédure } \\
\text { d'audition. }\end{array}$ \\
\hline $1484-1485$ & Scribe & Non & Main 1479 & $\begin{array}{l}\text { Compte assez soigné } \\
\text { très belle miniature } \\
\text { initiale (fig. } 4 \text { ) }\end{array}$ & $\begin{array}{l}2 \text { mains: } \\
\text { - sommes de fin } \\
\text { de chapitres } \\
\text { (auditeurs); } \\
\text { - corrections } \\
\text { (maître ou } \\
\text { scribe). }\end{array}$ \\
\hline $1486-1487$ & Scribe & Non & 1 main & $\begin{array}{l}\text { Compte soigné sans } \\
\text { lettrine ni initiales } \\
\text { travaillées }\end{array}$ & $\begin{array}{l}2 \text { mains } \\
\text { (auditeurs) } \\
\text { - sommes de fin } \\
\text { de chapitre; } \\
\text { - copie de la } \\
\text { commission des } \\
\text { auditeurs. }\end{array}$ \\
\hline
\end{tabular}




\begin{tabular}{|c|c|c|c|c|c|}
\hline $1487-1488$ & Scribe & Non & 1 main & & $\begin{array}{l}3 \text { mains : } \\
\text { - sommes } \\
\text { (auditeurs); } \\
\text { - corrections et } \\
\text { précisions } \\
\text { (maître ou } \\
\text { scribe); } \\
\text { - compte rendu } \\
\text { d'audition et } \\
\text { réception du } \\
\text { compte } \\
\text { (auditeurs). }\end{array}$ \\
\hline $1489-1490$ & Maître? & Non & 1 main & & $\begin{array}{l}1 \text { main } \\
\text { (auditeurs) } \\
\text { - sommes. }\end{array}$ \\
\hline $1491-1492$ & Maître? & Non & 1 main & & $\begin{array}{l}1 \text { main } \\
\text { (auditeurs) } \\
\text { - sommes. }\end{array}$ \\
\hline 1495-1496 & Maître? & Non & 1 main & Compte assez soigné & $\begin{array}{l}1 \text { main } \\
\text { (auditeurs) } \\
\text { - sommes }\end{array}$ \\
\hline $1496-1497$ & Maître? & Non & $\begin{array}{l}2 \text { mains } \\
-1 \text { pour essentiel } \\
\text { texte } \\
-1 \text { main p. } 321-332\end{array}$ & Compte assez soigné & $\begin{array}{l}1 \text { main } \\
\text { (auditeurs) } \\
\text { - sommes de fin } \\
\text { de recette. }\end{array}$ \\
\hline $1497-1498$ & Maître? & Non & & & \\
\hline 1498-1499 & Maître? & Non & & & \\
\hline
\end{tabular}

Tableau $\mathrm{n}^{\circ} 4$ : Nombre de comptes, de cahiers et de pages dans chaque volume des comptes hospitaliers

\begin{tabular}{|c|c|c|}
\hline Tome & Comptes inclus dans le volume & Nombre total de cahiers et de pages par volume \\
\hline Tome 1 & $\begin{array}{l}-1402-1403 \\
-1405-1406 \\
\text { - fragments 1431-1433 } \\
-1453-1454\end{array}$ & $\begin{array}{l}-13 \text { cahiers } \\
-380 \text { p. }\end{array}$ \\
\hline Tome 2 & $\begin{array}{l}-1454-1455 \\
-1456-1457\end{array}$ & $\begin{array}{l}-8 \text { cahiers } \\
-454 \text { p. }\end{array}$ \\
\hline Tome 3 & $\begin{array}{l}-1457-1458 \\
-1458-1459\end{array}$ & $\begin{array}{l}-9 \text { cahiers } \\
-382 p .\end{array}$ \\
\hline Tome 4 & $\begin{array}{l}-1460-1461 \\
-1462-1463\end{array}$ & $\begin{array}{l}-8 \text { cahiers } \\
-368 p .\end{array}$ \\
\hline Tome $5 / 1$ & $\begin{array}{l}-1467-1468 \\
-1468-1469\end{array}$ & $\begin{array}{l}-9 \text { cahiers } \\
-365 \text { p. }\end{array}$ \\
\hline
\end{tabular}




\begin{tabular}{|l|l|l|}
\hline Tome 5/2 & $-1470-1471$ & -6 cahiers \\
& $-1473-1474$ & $-468 \mathrm{p}$. \\
\hline Tome 6/1 & $-1475-1476$ & -5 cahiers \\
& -1479 & $-292 \mathrm{p}$. \\
\hline Tome 6/2 & $-1484-1485$ & -8 cahiers \\
& $-1486-1487$ & -398 p. \\
\hline Tome 6/3 & $-1487-1488$ & \\
& $-1488-1489$ & -8 cahiers \\
\hline & $-1491-1492$ & -335 p. \\
\hline Tome 7 & $-1495-1496$ & -7 cahiers \\
\hline & $-1496-1497$ & -644 p. \\
\hline
\end{tabular}

Tableau $\mathrm{n}^{\circ} 5$ : Les auditeurs désignés en 1487 , fonctions et responsabilités avant ou au moment de leur désignation

\begin{tabular}{|c|c|c|c|c|}
\hline $\begin{array}{ll}\text { Nom de } \\
\text { l'auditeur }\end{array}$ & $\begin{array}{l}\text { Fonctions tenues } \\
\text { au nom de la } \\
\text { communauté } \\
\text { bourgeoise }\end{array}$ & $\begin{array}{l}\text { Fonctions de } \\
\text { gestionnaire }\end{array}$ & $\begin{array}{l}\text { Fonctions ou } \\
\text { liens avec } \\
\text { l'hôtel-Dieu }\end{array}$ & $\begin{array}{l}\text { Métier et/ou } \\
\text { activités }\end{array}$ \\
\hline Jehan Beroult & $\begin{array}{lr}\text { Procureur } & \text { des } \\
\text { bourgeois } & \text { pour la } \\
\text { reddition } & \text { d'un } \\
\text { compte } & \text { d'impôt } \\
(1466) & \end{array}$ & $\begin{array}{l}\text { Trésorier de } \\
\text { Saint-Germain } \\
(1460-1462)\end{array}$ & $\begin{array}{l}\text { Auditeur des } \\
\text { comptes } \\
\text { hospitaliers } \\
\text { en } 1475\end{array}$ & clerc \\
\hline Arnoul Billart & \begin{tabular}{|l} 
Conseiller \\
gouverneur \\
procureur de la \\
ville en 1487
\end{tabular} & $\begin{array}{l}\text { Trésorier de } \\
\text { Saint-Germain } \\
(1477)\end{array}$ & $\begin{array}{l}\text { Auditeur des } \\
\text { comptes } \\
\text { hospitaliers } \\
\text { en } 1475\end{array}$ & \\
\hline Jehan Bunel & & & & $\begin{array}{l}\text { Lieutenant } \\
\text { général du } \\
\text { vicomte (1465) }\end{array}$ \\
\hline $\begin{array}{l}\text { Henry } \\
\text { de Bleseys }\end{array}$ & $\begin{array}{l}\text { Procureur des } \\
\text { bourgeois lors } \\
\text { d'une fondation de } \\
\text { messe à Saint- } \\
\text { Thomas (1483) }\end{array}$ & $\begin{array}{l}\text { Trésorier de } \\
\text { Saint-Martin } \\
\text { de } 1480 \text { à } 1482\end{array}$ & & \\
\hline
\end{tabular}




\begin{tabular}{|c|c|c|c|c|}
\hline Macé Hatesse & $\begin{array}{lr}\text { Procureur } & \text { des } \\
\text { bourgeois } & \text { pour la } \\
\text { reddition } & \text { d'un } \\
\text { compte } & \text { d'impôt } \\
(1466 \text { et } & 1472-1474)\end{array}$ & & & $\begin{array}{l}\text { Lieutenant du } \\
\text { vicomte } \\
\text { d'Argentan et } \\
\text { d'Exmes (1470); } \\
\text { lieutenant général } \\
\text { du bailli d'Alençon } \\
(1482)\end{array}$ \\
\hline Jehan Hauton & & $\begin{array}{ll}\text { Trésorier } & \text { de } \\
\text { Saint-Germain } & \\
(1454-1456) ; & \\
\text { conseiller } & \text { du } \\
\text { trésor } & \\
(1448-1450 & \text { et } \\
1462-1463) & \end{array}$ & $\begin{array}{l}\text { Désignation des } \\
\text { auditeurs (1475) } \\
\text { et auditeur des } \\
\text { comptes } \\
\text { hospitaliers } \\
(1475)\end{array}$ & $\begin{array}{l}\text { Gouverneur de la } \\
\text { ville d'Argentan } \\
(1454)\end{array}$ \\
\hline Gilles de La Pallu & & & & Écuyer \\
\hline Berthelot Loison & $?$ & $?$ & ? & $?$ \\
\hline $\begin{array}{l}\text { Guillaume } \\
\text { Noël l'aîné }\end{array}$ & & & $\begin{array}{l}\text { Témoin de la } \\
\text { fondation d'une } \\
\text { messe par le } \\
\text { maître (1483) }\end{array}$ & \\
\hline Edouard Nollet & & & $\begin{array}{l}\text { Témoin de la } \\
\text { fondation d'une } \\
\text { messe par le } \\
\text { maître (1483) }\end{array}$ & \begin{tabular}{|l} 
Conseiller \\
gouverneur et \\
procureur de la \\
ville d'Argentan \\
$(1487)$
\end{tabular} \\
\hline Romain Pigache & $\begin{array}{lr}\text { Procureur } & \text { des } \\
\text { habitants de } & \text { la } \\
\text { vicomté d'Argentan } \\
\text { et d'Exmes } & \text { aux } \\
\text { Etats de Normandie } \\
(1476) & \\
\text { Procureur } & \text { des } \\
\text { bourgeois } & \text { pour } \\
\text { l'audition } & \text { des } \\
\text { comptes de } & \text { Saint- } \\
\text { Germain } & \\
(1462-1463) & \end{array}$ & $\begin{array}{llr}\text { Responsable } & \text { de } \\
\text { la } & \text { recette } & \text { et } \\
\text { dépense } & \text { de } \\
\text { l'impôt } & \text { urbain } \\
(1452) & \end{array}$ & $\begin{array}{l}\text { Auditeur des } \\
\text { comptes (1470 à } \\
1480, \quad 1484-85, \\
1486-1487,1489)\end{array}$ & $\begin{array}{l}\text { Procureur et clerc } \\
\text { du vicomte } \\
\text { d'Argentan et } \\
\text { Exmes (1446 } \\
1447) \text {, receveur de } \\
\text { la vicomté } \\
(1454-1494)\end{array}$ \\
\hline Guy Pitart & & & $\begin{array}{l}\text { Témoin de la } \\
\text { fondation d'une } \\
\text { messe par le } \\
\text { maître (1483) }\end{array}$ & Écuyer \\
\hline Pierres Pitart & $?$ & $?$ & $?$ & $?$ \\
\hline Perrinot Sayecte & $\begin{array}{l}\text { Procureur des } \\
\text { bourgeois en } 1463\end{array}$ & $\begin{array}{l}\text { Trésorier de } \\
\text { Saint-Germain } \\
(1463-1465)\end{array}$ & $\begin{array}{l}\text { Désignation des } \\
\text { auditeurs (1475) }\end{array}$ & \begin{tabular}{|l} 
lonseiller \\
gouverneur et \\
procureur de la \\
ville d'Argentan \\
$(1487)$
\end{tabular} \\
\hline
\end{tabular}


Fig. 1 : Première page du $6^{\mathrm{e}}$ compte de Charles Lemesnagier (1454-1455)

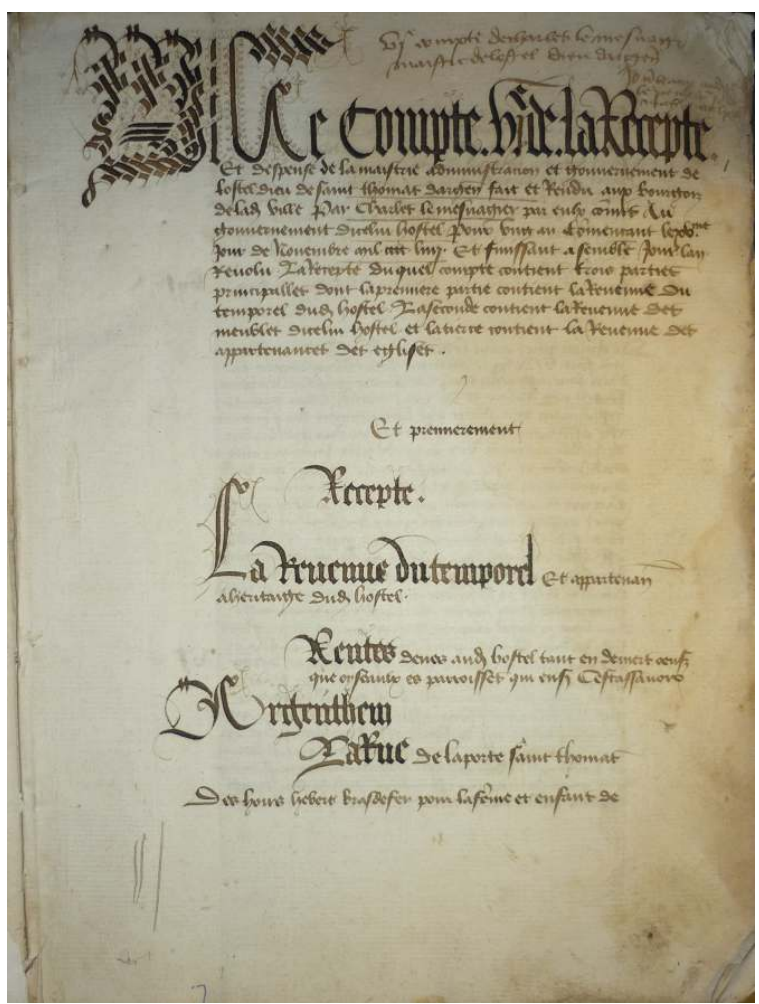

La belle présentation du compte, le soin apporté à l'écriture et à la mise en page montre la volonté de reprise en main de l'hôtel-Dieu ainsi que de ses biens et droits, par le nouveau maître, après la période troublée de l'occupation anglaise.

Alençon, Arch. dép. Orne, H dépôt 2, tome 2, p. 1, (cliché M.-A. Moulin, autorisation Arch. dép. Orne) 
Fig. 2 : Première page du $22^{\mathrm{e}}$ compte de Charles Lemesnagier (1470-1471).

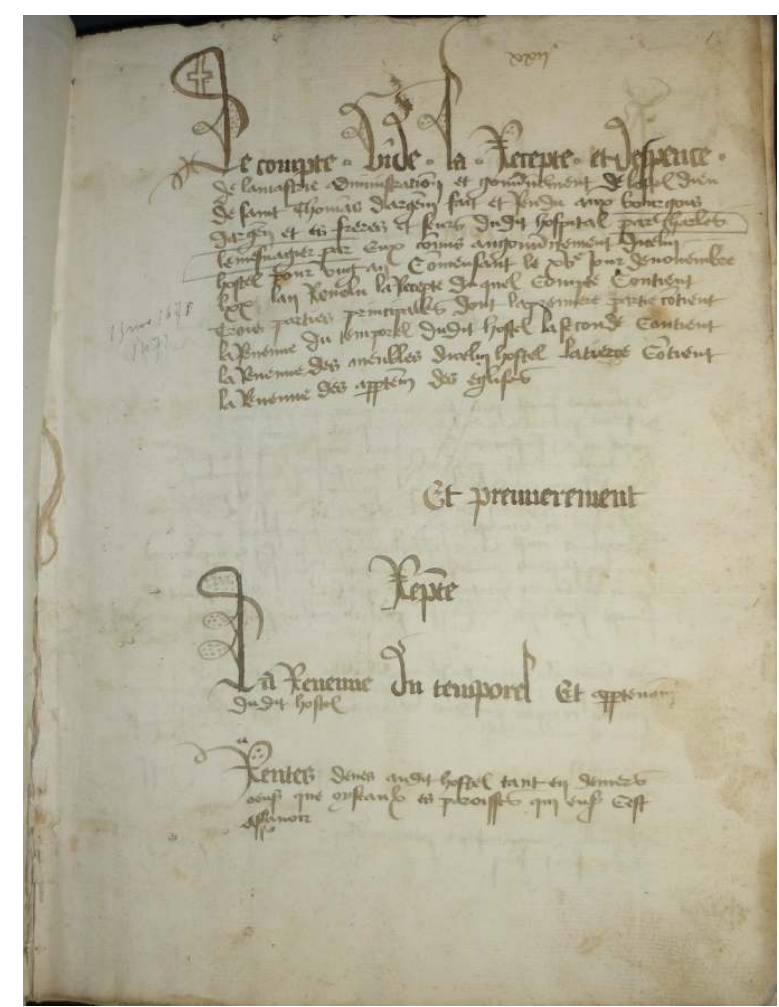

Le paragraphe initial porte rappel du mandat du maître désigné par les bourgeois, indique les termes de son administration et fait l'annonce des chapitres des recettes et des dépenses qui sont détaillés dans le compte.

Alençon, Arch. dép. Orne, H dépôt 2, tome 5/2, p. 1, (cliché M.-A. Moulin, autorisation Arch. dép. Orne) 
Fig. 3 : Première page du compte de Charles Lemesnagier de 1474-1475.

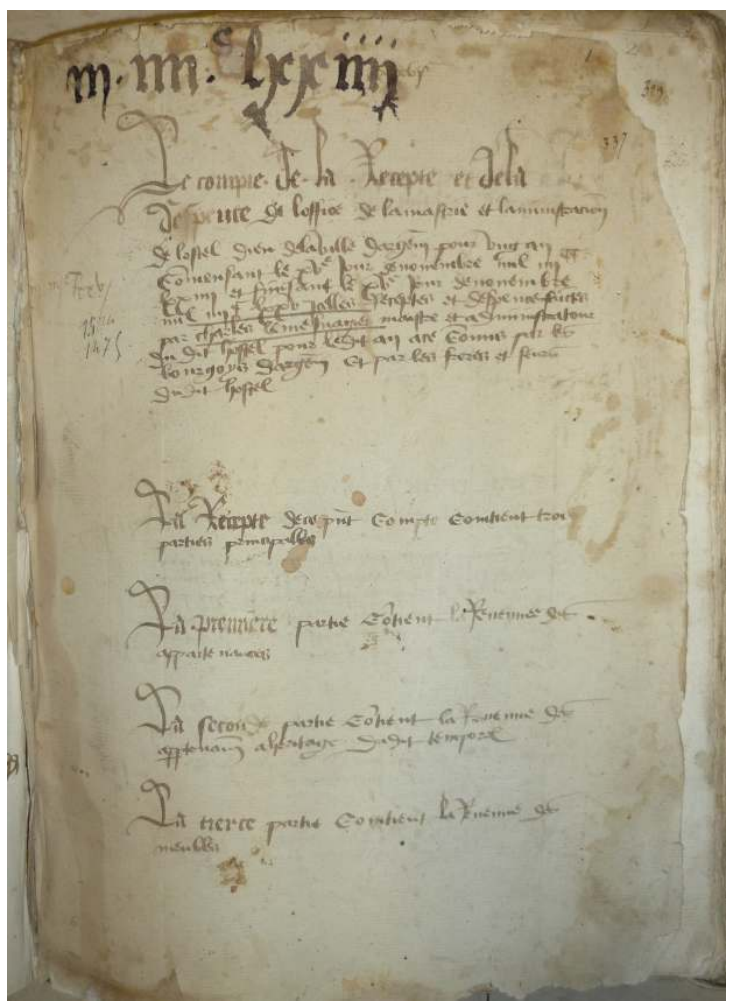

Cette page montre le peu de soin apporté à la rédaction et à la présentation des comptes en fin d'administration de ce maître, alors même que ses premiers comptes se distinguent par leur qualité Alençon, Arch. dép. Orne, H dépôt 2, tome 5/2, p. 337, (cliché M.-A. Moulin, autorisation Arch. dép. Orne) 
Fig. 4 : Première page du compte de Macé Gaultier pour l'exercice de 1484-1485, orné d'une belle représentation de saint Thomas archevêque de Cantorbéry, saint patron de l'hôtel-Dieu argentanais. Cette miniature, particulièrement soignée dans un compte par ailleurs assez simple, est sans équivalent dans la comptabilité hospitalière

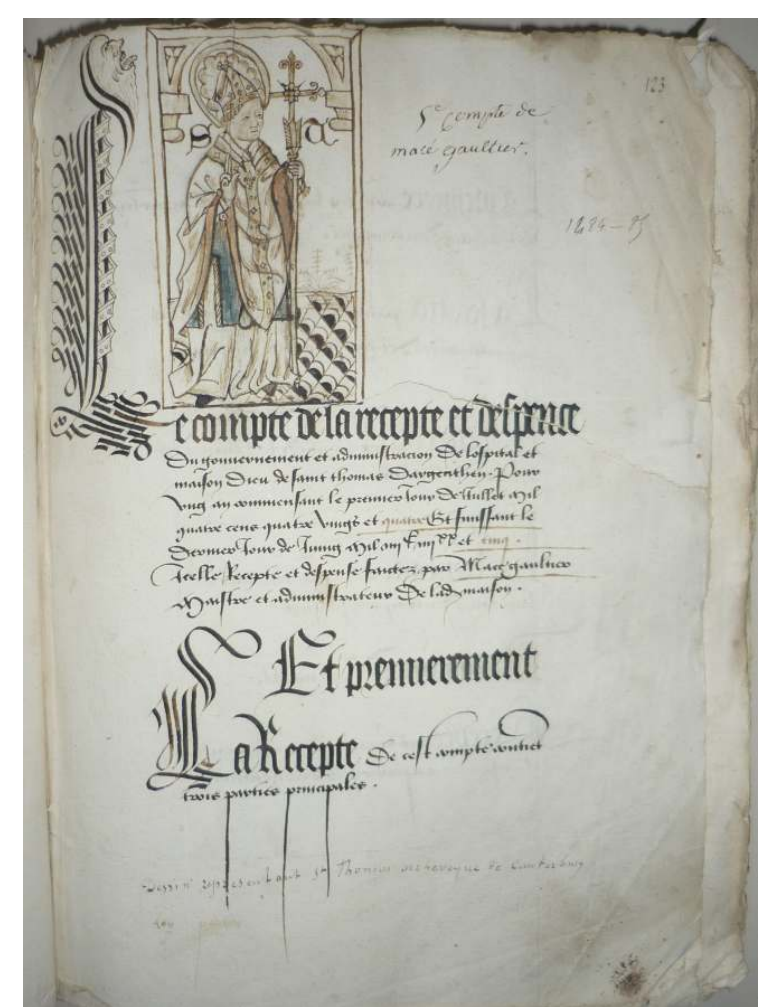

Alençon, Arch. dép. Orne, H dépôt 2, tome 6/2, p. 123, (cliché M.-A. Moulin, autorisation Arch dép. Orne) 
Fig. 5 : Fin du compte de 1454-1455 (Charles Lemesnagier) faisant le récapitulatif des recettes et dépenses engagées en cette année de compte.

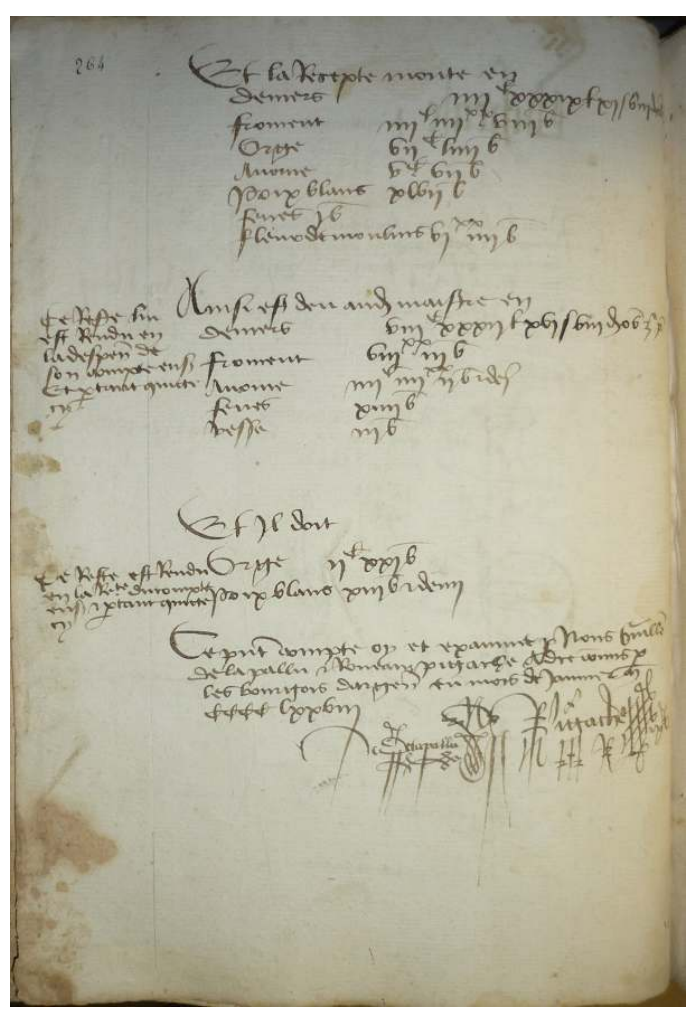

Le bas de page porte mention de la réception du compte en janvier 1479. Pour attester que le maître est quitte de son administration, les auditeurs, dont l'identité est rappelée, ont ajouté leur paraphe.

Alençon, Arch. dép. Orne, H dépôt 2, tome 2, p 264, (cliché M.-A. Moulin, autorisation Arch. dép. Orne)

\section{NOTES}

1. La fondation d'hôtels-Dieu par des bourgeois n'est pas inhabituelle dans le domaine normand et leur gestion est alors confiée à des maîtres représentant les bourgeois [FAZY, 1913, p. 190 ; LE CACHEUX, 1895, p. 8].

2. Les personnes qui se donnent ainsi à l'administration hospitalière sont désignées comme des « donnés » ou plus souvent « condonnés ».

3. IMBERT, 1982, p. 73.

4. Alençon, Arch. dép. Orne, $\mathrm{H}$ dépôt 2/253, F 1 - 1374, 16 juillet (copie collationnée à l'original 1576, 3 novembre) : acte par lequel Nicole Le Petit est déclaré quitte de son administration.

5. La somme de 300 livres est effectivement exigée de ce maître avant qu'il ne soit déclaré quitte de son administration [Alençon, Arch. dép. Orne, H dépôt 2/253, F 2 - 1397, 2 juillet : contrat par lequel Hamelin le Boucher, maitre et administrateur de Saint-Thomas, pour demeurer quitte de son administration, s'oblige à payer 300 livres].

6. 11 s'agit des comptes de 1402-1403 et de 1405-1406. Le compte de 1407 n'est connu que par des extraits copiés sur l'original au XVII ${ }^{\mathrm{e}}$ siècle (voir tableau de présentation des comptes en fin d'article).

7. Les années pour lesquelles les comptes hospitaliers sont aujourd'hui conservés aux Archives départementales de l'Orne sont les suivantes : 1521-1552 ; 1527-1532 ; 1538-1541 ; 1545-1546 ; 1548-1449 ; 1551-1629 série quasiment ininterrompue avec seulement l'année 1582 manquante ; 
$1636-1657 ; 1660 ; 1662-1663 ; 1665-1692 ; 1695-1712 ; 1723-1790$. À ma connaissance, cette formidable série moderne n'a jusqu'à présent pas fait l'objet d'une étude poussée.

8. Lors de la chevauchée du duc de Lancastre en 1356, le château d'Argentan est attaqué et partiellement détruit. Les titres les plus importants de l'hôtel-Dieu y étaient conservés et certains d'entre eux ont alors disparu. À la suite de cet incident, les actes hospitaliers sont conservés à l'hôpital même, dans la chambre du maître [COURTEILLES, 1693-1694, p. 75].

9. Alençon, Arch. dép. Orne, H dépôt 2, non coté - 1570-1573 : «Inventaire des lectres et ensaignemens de la maison dieu Sainct Thomas d'Argentan trouvés aux casses et coffres de ladite maison par vénérable personne maistre Thomas le Coifferel prebstre ». Le registre ne fait mention que des comptes de recette et de dépense de Le Coifferel des années 1570-1574 et des 6 comptes de Thibault Biard (1565-1570).

10. Alençon, Arch. dép. Orne, H dépôt 2 B 9 - 1616-1620 : "Mémoires et extraits des comptes par lesquels il paroist que les loges de la venelle des champs et de la porte Saint-Thomas ont été basties aux dépends et sur le fond de la maison Dieu en 1616 et vers 1620 »; H dépôt 2 B 23 - 1688 : " pièces d'écriture qui sont mémoires, requestes, copies informes d'actes et sentences et extraits de comptes concernant [les] droits de foire "; H dépôt 2 B 43 - s. d. : extraits des comptes de 1402 et 1405 pour justifier les droits dont l'hôtel-Dieu jouit sur plusieurs moulins de la région ; H dépôt 2 B 6 - 1777 : extrait du compte de 1405; H dépôt 2 B 3 - 1835 : extrait du compte de 1405 justifiant de la propriété d'une maison à Argentan. L'abbaye Notre-Dame d'Almenêches a elle aussi recours à ces archives hospitalières [Alençon, Arch. dép. Orne, H 3589, nº 6-1631 : copies des « piesses consernant la chapelle Notre-Dame d'Argentan qui ont esté produites par le sieur des Pantoullères ", la liasse contient des extraits des comptes de 1402-1403, 1453-1454 et 1463-1464].

11. Alençon, Arch. dép. Orne, H dépôt 2 C 28 : extraits des comptes de 1579 à 1587 contenant les charges du chapelain de Notre-Dame de Bonvouloir.

12. Cette série de procès fait suite à la décision de l'abbesse d'installer les moniales en ville dans une église sur laquelle les habitants prétendent avoir des droits [Alençon, Arch. dép. Orne, H 3589, nº 3 - 1630-1631: copie des pièces produites par l'abbaye Notre-Dame d'Almenêches contre Messieurs de La Pallu dont des copies des anciens comptes hospitaliers de 1407-1408; H $3589, \mathrm{n}^{\circ} 4$ - 1631 : extrait du compte de 1417-1418].

13. COURTEILLES, 1693-1694, p. 140 ; ms 10, p. 71 ; ms 11, p. 85.

14. Lors d'une attaque de la ville par les protestants menés en 1568 par Gabriel de Montgommery, les archives du trésor de Saint-Martin d'Argentan sont en grande partie détruites. Lors d'autres épisodes des guerres de Religion, les archives et les biens de la paroisse Saint-Germain et des dominicains de la ville subissent également des dommages importants [LAURENT, 1859, p. 66-69].

15. Il faut souligner que lors de la constitution de la série $\mathrm{H}$, l'archiviste n'emporte aucun original hors de l'enceinte hospitalière. Des actes ont été retrouvés à son domicile après son décès et ont été réintégrés dans les Archives départementales mais aucun ne concernait l'hôpital argentanais [Alençon, Arch. dép. Orne, C supplément non coté - «Documents trouvés chez M. Louis Duval après son décès et déposés à la SHAO, réintégrés aux Archives départementales en décembre $1926 »]$.

16. LAURENT, 1859, p. 165.

17. COURTEILLES, 1693-1694, p.140. Ces comptes dont Courteilles se sert dans son étude sur l'hôpital sont aujourd'hui disparus.

18. Les textes de l'après-guerre insistent sur l'état de délabrement des bâtiments hospitaliers et la baisse significative des revenus de Saint-Thomas. Les dons - en nature et en argent - consentis par le duc d'Alençon, seigneur d'Argentan, au profit de l'institution prouvent bien la nécessité de remettre à flot ses finances. Sur ce sujet, voir MOULIN, 2007.

19. Alençon, Arch. dép. Orne, $\mathrm{H}$ dépôt $2 / 253$, boite 3, F 4 : copie collationnée à l'original de l'arrêt du Parlement de Normandie destituant maître Thomas Hungtynton, prêtre anglais, de 
l'administration de l'hôtel-Dieu Saint-Thomas et rétablissant les bourgeois d'Argentan dans leur droit de désignation.

20. La rédaction des comptes hospitaliers du début $d u X V^{e}$ siècle semble s'être toujours faite sur parchemin ainsi que le laisse penser une copie partielle d'un compte de 1407-1408, réalisée au $\mathrm{XVI}^{\mathrm{e}}$ siècle. La copie collationnée à l'original stipule, en introduction, qu'il s'agit « du compte en parchemin rendu par Guillaume Nicolle, prêtre, administrateur de laditte maison [Dieu] pour l'année commençant le $1^{e r}$ jour de mars 1407 ». Ce même acte porte copie d'extraits d'un compte rendu en 1417 lui aussi rédigé sur parchemin «autre extrait de parchemin » [Alençon, Arch. dép. Orne, H 3589, pièce $n^{\circ} 6$ : Extraits du compte de Guillaume Nicolle, prêtre, maître et administrateur de l'hôtel-Dieu Saint-Thomas, rendu le $1^{\mathrm{er}}$ mars 1407 (a.st) et extrait d'un compte rendu en 1417]. Aucune source ou historiographie locale ne fait a contrario mention de comptes tenus sur papier avant le compte de 1454-1455.

21. Alençon, Arch. dép. Orne, par exemple : H dépôt 2 B 13 - acte de vente, 1512.

22. L'absence de mention d'achat de peaux ou de parchemin déjà prêt va dans le sens d'une fabrication du parchemin au sein de l'hôtel-Dieu. Cette hypothèse est d'autant plus probable que l'institution possède plusieurs troupeaux d'ovins et de bovins qui alimentent ses réserves en viande et en cuirs [MOULIN, 2007, tome 1].

23. Les comptes de la vicomté voisine d'Exmes, aujourd'hui conservés à la Médiathèque d'Argentan, sont encore rédigés sur parchemin en 1445-1447.

24. De nombreux moulins à blé ont été construits sur les cours d'eau d'Argentan et de ses environs dès le XII ${ }^{\mathrm{e}}$ siècle. Au XIV siècle, des moulins à tan et des moulins foulons $\mathrm{y}$ sont également établis. Mais si l'existence de moulins à papier n'y est pas prouvée par les sources documentaires, elle apparaît de ce fait très probable [sur l'économie urbaine et du plat-pays, voir MOULIN, 2007, tome 1].

25. C'est dans le compte de 1460 qu'il est fait mention de l'achat de six mains de papier auprès de la veuve Thibault de La Lande.

26. Sur les différents types de filigrane de licorne et plus particulièrement sur les filigranes de licorne simple, voir BRIQUET, 2000, vol.3, p. 523 et suivantes. Dans son travail, Briquet semble n'avoir examiné aucun papier argentanais ce qui pourrait expliquer l'absence des filigranes locaux dans son répertoire.

27. Les dimensions des licornes examinées par Briquet oscillent de 59 à $84 \mathrm{~mm}$ de haut sur 112 à $129 \mathrm{~mm}$ de large.

28. Dans sa thèse et dans des articles consacrés au trésor de Saint-Germain d'Argentan [VÉRITÉ, 1990, 1991, 2008], Isabelle Vérité mentionne le filigrane de licorne, mais elle n'en indique pas les dimensions, ce qui rend toute comparaison hasardeuse [VÉRITÉ, 1990, livre 2, p. 4. La licorne n'est, cependant, pas le seul filigrane relevé dans la comptabilité de Saint-Germain].

29. BRIQUET, 2000, vol. 3, p. 519.

30. Ibid., vol. 1, p. 121.

31. Ibid.

32. Ibid., vol. 4, p. 685-686.

33. Ibid., vol. 1, p. 4.

34. Alençon, Arch. dép. Orne, Comptes de la terre de Montgommery, A Montg. XIX ${ }^{5}$ - compte de la terre de Montgommery - Saint Michel 1404 - Saint Michel 1405. Ce registre mesure $220 \mathrm{~mm} \mathrm{~L} \mathrm{x}$ $300 \mathrm{~mm} \mathrm{H}$.

35. Argentan, Méd., ms 17, 1445-1447, fol. 88 et fol. 139v.

36. En 1452, Pigache est désigné responsable de la recette et dépense de la «maille ", impôt levé sur les boissons vendues en ville pour le pavage des portes de la ville [Alençon, Arch. dép. Orne, H $3589, \mathrm{n}^{\circ} 7-1453,16$ juillet].

37. Argentan, Méd., ms 17, 1445-1447, fol. 139v. 
38. Paris, Arch. nat., KK 648, fol. 36, n 33 - 1476, 15 décembre.

39. Les comptes de l'abbaye voisine de Notre-Dame de Silly, qui ont gardé leur reliure médiévale, sont ainsi rassemblés en registres dotés d'une couverture de parchemin épais, par exemple : Alençon, Arch. dép. Orne, H 1121 - comptes de l'abbaye de 1438-1442 ; H 1116 - comptes de l'année 1499.

40. Bury est désigné maître de l'hôtel-Dieu par le roi d'Angleterre en 1435, mandat qui n'est pas reconnu par les habitants et qui n'est pas renouvelé par la suite, et Jehan Duval cesse d'exercer ses fonctions de vicomte de la ville en 1439 pour devenir lieutenant du bailli d'Alençon [MouliN, 2007, tome 1, p. 92 ; p. 228-229].

41. Argentan, Méd., ms 17 - 1445-1447. Les comptes de ces trois années successives ont été réunis en un seul registre.

42. Alençon, Arch. dép. Orne, $\mathrm{H}$ 3589, pièce $\mathrm{n}^{\circ} 6$ - copie collationnée à l'original en 1631.

43. Des pages sont ainsi manquantes dans le compte de 1456-1457 [H dépôt 2, tome 2, compte de 1456-1457, feuillet manquant entre les pages 412 et 413], dans le compte de 1458 [H dépôt 2 , tome 4, compte de 1458-1459, deux feuillets découpés entre les pages 352 et 353], dans le compte de 1460 [H dépôt 2, tome 4, compte de 1460-1461, des feuillets ont été déchirés entre les pages 182-183, une partie de la page 183, trois pages manquent entre les p.184-185], dans le compte de 1461 [H dépôt 2, tome 4, compte de 1461-1462, plusieurs pages déchirées entre les p. 241-242], dans le compte de 1474-1475 [H dépôt 2, tome 5/2, compte de 1474-1475, p. 348-349, p. 350-351, p. 356-357, p. 390-391, p. 396-397 et les 4 derniers feuillets du compte ont disparu]. Les dernières pages du compte de 1473 ont été déchirées [H dépôt 2, tome 5/2, compte de 1473-1474]. 44. De telles récupérations de papier ont pu être constatées pour les comptes de 1474, de 1479 et de 1487 [Alençon, Arch. dép. Orne, tome 5/2, compte de 1474-1475, plusieurs pages soigneusement découpées entre les pages 348-349, 350-351 et 356-357, de plus deux feuillets ont été découpés entre les pages 396 et 397 ; tome 6/2, compte de 1479, une page manquante entre les pages 88-89; tome 6/3, compte de 1487-1488, page découpée entre les pages 130-131].

45. Dans le compte de 1467, apparaît ce qui semble être un exercice de copie «Je dis au pas de Rome va dire à saint Martin » [H dépôt 2, tome 5/1, compte de 1467-1468, p. 59]. Dans ce même compte, plusieurs paragraphes sont recopiés à la suite du texte médiéval [ibid. p. 48-49, p. 58, p. 59 et 63, p. 115, p. 118-119, p. 121].

46. Des essais de plume sont visibles dans les comptes de 1453 [H dépôt 2, tome 1, compte de 1453-1454, p. 307], 1456 [H dépôt 2, tome 2, compte de 1456-1457, p. 420 et 429] et 1458 [H dépôt 2, tome 4, compte de 1458-1459, p. 344]. Ce sont plusieurs essais de paraphe qui sont visibles dans le compte de 1467 [H dépôt 2, tome 5/1, compte de 1467-1468, p. 122] et dans le compte de 1475 [H dépôt 2, tome 6/1, compte de 1475-1476, p. 76 et p. 283].

47. Un extrait d'acte notarié est ainsi noté dans le compte de 1458: «Je Mary Burnel maistre et administrateur de la maison dieu d'Argentan confesse avoir eu et receu de Maurice James bourgoys la somme de vingt sous " [H dépôt 2, tome 4, compte de 1458-1459, p. 298]. L'indication du nom du maître permet de dater ce brouillon des années 1554-1559, période de son administration. Le compte de 1467 porte plusieurs extraits ou essais d'actes administratifs tels un courrier adressé au vicomte [H dépôt 2, tome 5/1, compte de 1467-1468, p. 73] et un compterendu de plaids [ibid. p. 99].

48. Les premières traces de foliotation apparaissent sur les vingt premiers feuillets du compte de 1405-1406. La pagination de 1831 s'est superposée à cette foliotation initiale. Les autres comptes foliotés au Moyen Âge sont ceux de 1453-1454, totalement numéroté, de 1468-1469 (jusqu'au 29e feuillet), de 1474-1475 (jusqu'au folio 9) et de 1475-1476 (jusqu'au feuillet 19).

49. Il s'agit du compte de 1453-1454. Peut-être faut-il voir dans cet ajout une volonté de clarté et d'organisation de la source comptable à la suite de la guerre de cent ans et d'une longue période d'instabilité pour l'hôtel-Dieu.

50. C'est cette pagination contemporaine qui a été retenue pour les références aux comptes. 
51. Alençon, Arch. dép. Orne, $\mathrm{H}$ dépôt 2, 253 - 1449, 15 novembre : contrat passé devant les tabellions d'Argentan par lequel Charles Lemesnagier est nommé maître et administrateur de l'hôtel-Dieu Saint-Thomas d'Argentan (copie).

52. Alençon, Arch. dép. Orne, $\mathrm{H}$ dépôt 2 , tome 6/1, compte de 1479, p. 1 : «le derrain compte dudit deffunct [Charles Lemesnagier] finissant le $15^{\mathrm{e}}$ jour de novembre 1479 seulement afin que les comptes dudit hostel se commencent doresenavant audit premier jour de juing juillet ».

53. Alençon, Arch. dép. Orne, H dépôt 2, 253 - 1495, 21 juillet : contrat passé devant les tabellions d'Argentan par lequel Gautier Chéradames est nommé maître et administrateur de l'hôtel-Dieu Saint-Thomas (copie).

54. Les comptes de 1453-1454 sont entendus le 22 mai 1477, ceux de 1454-1455 le sont en janvier 1479 et les comptes de 1457 à 1462 sont présentés en mars 1479.

55. VENTURINI, 1988, p. 80. L'auteur note un « écart sensible entre la date de reddition du compte (9 décembre 1343) et la fin de la période couverte (31 août 1341)» et signale qu'à l'époque un tel écart « n'a rien d'anormal ».

56. Voir tableau 1.

57. Le mandat des auditeurs est le plus souvent rappelé sous la forme suivante: «ce present compte ouy et examiné par nous ", formule suivie de l'identité des auditeurs puis des termes « ad ce commis par les bourgois [sic] d'Argenthen » puis de la date de l'audition. La mention inscrite en haut de la première page des comptes de 1453-1454 et de 1454-1455 diffère puisqu'il y est clairement indiqué «Présenté aux auditeurs » ainsi que la date de l'audition.

58. Le total de la dépense de Saint-Thomas ne peut effectivement se faire qu'à l'issue de l'audition puisque les frais qu'elle engendre sont ponctionnés sur la recette de la même année.

59. La durée de l'audition, entre deux et quatre jours, est alors spécifiée puisqu'elle fait varier le montant des frais.

60. Les comptes se concluent sur les paraphes de l'ensemble des auditeurs. Seul le compte de 1460 fait exception puisque seul l'un des auditeurs a signé le registre.

61. Il arrive cependant que ces feuillets aient fait l'objet d'une récupération ultérieure et aient alors été soigneusement découpés.

62. Alençon, Arch. dép. Orne, tome 3, compte de 1458-1459, p. 373.

63. Ibid., tome 6/2, compte de 1479 , p. 31 et p. 103.

64. Ibid., p. 103. C'est ce que laisse entendre la décision d'imputer au compte ordinaire du maître l'achat de drap pour lui et sa femme et de retrancher cette dépense du compte hospitalier: « ceste partie est comptée et alloué ou compte ordinaire dudit maistre commensant le premier de juillet $1480 »$.

65. Alençon, Arch. dép. Orne, H dépôt 2, tome 6/3, compte de 1488-1489, p. 214 : « lequel revenu est compté ou journal du maistre par les sepmaines et icy abregié par les moys ».

66. Ibid., tome 6/2, compte de 1479, p. 103.

67. C'est par exemple le cas en 1458, le scribe est alors rémunéré pour « la façon et escripture de ce present compte et des journaux et pappiers ordinaires » [Alençon, Arch. dép. Orne, tome 3, compte de 1458-1459, p. 373].

68. Argentan, Méd., ms 40 : copie collationnée du contrat passé devant Jehan Biart tabellion à la demande des bourgeois pour établir Charles Lemesnagier maître et administrateur de SaintThomas à la condition qu'il apporte en grains et en bétail l'équivalent de 100 livres et qu'il fasse don de 200 livres à employer pour le bien de la maison-Dieu.

69. Alençon, Arch. dép. Orne, tome 7, compte de 1495-1496, p. 111. Un paragraphe spécifique est consacré à cette aide qu'apporte l'hôpital à l'une des deux églises paroissiales de la ville : "Autre despence a cause de l'église de Sainct Germain d'Argenthen ». La somme versée s'élève alors à vingt livres.

70. Alençon, Arch. dép. Orne, $\mathrm{H}$ dépôt 2/253, F 1 - 1374, 16 juillet (copie collationnée à l'original - 1576, 3 novembre) : acte par lequel Nicole Le Petit est déclaré quitte de son administration. 
71. Dans la ville comme dans un rayon d'une vingtaine de kilomètres, l'hôpital est propriétaire de nombreux terrains, jardins, maisons et hébergements.

72. Ces droits consistent en un droit à prendre une charrette de bois à brûler et une charrette de bois à bâtir par jour en forêt et du droit de panage de soixante porcs. La valeur de ces droits est estimée dans le chapitre des dépenses même si l'hôtel-Dieu n'en fait que rarement commerce.

73. C'est notamment le cas du compte de 1453-1454 écrit par une seule main et qui porte un nombre important de précisions et des ratures mais ne comporte aucun espace laissé vierge.

74. La procédure de nomination est bien connue. Les bourgeois, habituellement en petit nombre, se réunissent dans un premier temps pour désigner un nombre variable de bourgeois constituants qui, à leur tour, désignent le maître et administrateur de Saint-Thomas. Le maître est quelquefois l'un de ces bourgeois constituants qui, dans les faits, représentent un groupe restreint possédant une forte influence sur les destinées de l'institution charitable.

75. C'est à cet effet que le maître et sa femme, s'il est marié, disposent d'un logement au sein même de l'enceinte hospitalière.

76. Alençon, Arch. dép. Orne, H dépôt 2/253, nº 5 (F 5) - 1449, 15 novembre.

77. Ibid.

78. Ibid., $\mathrm{H}$ dépôt $2 / 231$.

79. C'est une mention marginale du compte de 1479 qui permet d'affirmer que le journal du maître recense toutes ses dépenses, personnelles ou pour l'hôpital, et que toutes ne sont pas retenues dans le compte définitif. À propos de drap acheté pour le maître et sa femme, il est stipulé que : «Ceste partie est comptee et allouee ou compte ordinaire dudit maistre comensant le premier jour de juillet $1480 »$.

80. Alençon, Arch. dép. Orne, H dépôt 2 C 32 - 1585-1586 : cahier des « dépenses quotidiennes pour aumônes faites aux pauvres et passans ».

81. Versés au XIX ${ }^{\mathrm{e}}$ siècle aux Archives départementales, ces actes sont intégrés dans le fond de l'hôtel-Dieu en série H dépôt 2.

82. Les travaux de construction menés pendant la période aux édifices de Saint-Thomas en sont un exemple flagrant. Aucun mandement de travaux ni aucune quittance n'a été conservée au contraire de sites comme le château ou les fortifications urbaines ; sur ce sujet, voir Moulin, 2007.

83. Alençon, Arch. dép. Orne, $H$ dépôt 2, tome 6/2, compte de la Saint-Michel 1479, p. 11.

84. Ibid., p. 21 et p. 30. Des précisions semblables sont portées aux pages 25 et 29.

85. Ibid., p. 17.

86. Ibid., p. 31 : à propos d'une rente due par les enfants de Jehan Gacé, les auditeurs exigent du maître qu'il «face diligence d'en recouvrir payement et en responde en ses comptes ordinaires »; p. $38:$ « face diligence le maistre et en responde ».

87. Ibid., p. 50 : Bardin Lefort, redevable d'une rente à Tournai, « soit contraint par le maistre a compter et payer ce qu'il doit »; p. 52 : « soit faicte contrainte et en responde le maistre ». Il reste cependant possible au maître de démontrer son incapacité à percevoir des sommes dues à l'hôtelDieu comme le prouve une note marginale à la page 103 : «De la Dame du chastel d'Almenesches trouvé en reste 10 sous dont le maistre n'a sceu trouver les lectres et contiennent les comptes anciens que ladite rente est assise sur le moulin dudit lieu d'Aulmenesches, pour ce néant ».

88. Alençon, Arch. dép. Orne, $\mathrm{H}$ dépôt $2 / 226$ : Inventaire des lettres, titres et revenus de l'hôtelDieu d'Argentan - avril 1555.

89. La nature particulière de ces fragments ne fait pas de doute et ils ne sauraient être confondus avec des comptes définitifs. Au contraire d'un compte définitif, les biens sur lesquels portent les rentes n'y sont pas détaillés, seuls apparaissent les noms des tenants et les sommes ainsi que le terme de versement concerné. Cependant, le même ordre géographique préside à la présentation des rentes que dans les comptes définitifs. Autre différence notable, les gages du maître (fol. 7) ne sont jamais portés aux comptes définitifs, mais ils sont ici indiqués. 
90. Les précisions sont apportées dans le corps du texte, notamment dans les espaces laissés vierges dans les comptes pré-rédigés. Elles se distinguent à la fois par le changement d'écriture et le changement d'encre. Elles sont a priori attribuables au maître, aucune dépense n'étant relative à cet amendement du texte pré-rédigé.

91. Les sommes de fin de paragraphe sont portées en marge et les sommes des quatre principaux chapitres sont écrites à la suite du texte dans un espace laissé vacant à cet effet. Toutes les sommes sont le fait des auditeurs de même que le rajout des frais d'audition et l'éventuelle copie de leur commission.

92. Les ratures sont attribuables à la personne chargée de compléter le compte avant son audition, à savoir, très probablement, le maître lui-même.

93. Le scribe n'ayant pu être identifié, il est distingué par l'année du $1^{\mathrm{er}}$ exercice de compte qu'il a rédigé.

\section{RÉSUMÉS}

Les Archives départementales de l'Orne conservent en série $\mathrm{H}$ dépôt 2, l'ensemble des comptes de l'hôtel-Dieu Saint-Thomas d'Argentan. Pour la période médiévale, la comptabilité hospitalière couvre - très inégalement - les années 1402-1499. Disposer d'une série sur une période aussi longue permet, d'une part, de dégager les grands principes auxquels obéit une comptabilité : mode de tenue du compte, structure d'un compte type, termes d'ouverture et de clôture des comptes, règles de présentation et, d'autre part, de percevoir l'évolution séculaire de la comptabilité.

The archive service of the Orne department conserves in the series $\mathrm{H}$ deposit 2, a series of accounts from the Hospital of Saint-Thomas d'Argentan. For the medieval period hospital accounting covers - very unequally - the years between 1402 and 1499. The possession of a series covering such a long period has brought to light the wider principles to which the accountancy obeyed: the modalities of account keeping, the structure of a model account, the terms of opening and closing accounts, the rules of presentation; and on the other hand perceive the evolution of secular accountancy.

\section{INDEX}

Mots-clés : Argentan, hôtel-Dieu, maître et administrateur, comptabilité, gestion, audition des comptes, scribe, parchemin, papier, filigrane, procédure comptable

Keywords : Argentan, hospital, master and administrator, accountancy, scribe, parchment, paper, filigree, account procedure

\section{AUTEUR}

\section{MARIE-ANNE MOULIN}

CRAHAM

UMR 6273 UCBN/CNRS 